\title{
A PRIORI ESTIMATES FOR MANY-BODY HAMILTONIAN EVOLUTION OF INTERACTING BOSON SYSTEM
}

\author{
MANOUSSOS G. GRILLAKIS \\ Department of Mathematics, University of Maryland \\ College Park, Maryland 20742, USA \\ mng@math.umd.edu \\ DIONISIOS MARGETIS \\ Department of Mathematics, University of Maryland \\ College Park, Maryland 20742, USA \\ and \\ Institute for Physical Science and Technology, University of Maryland \\ College Park, Maryland 20742, USA \\ dio@math.umd.edu
}

Received 28 Dec. 2007

Accepted 22 May 2008

Communicated by Philippe G. LeFloch

\begin{abstract}
We study the evolution of a many-particle system whose wave function obeys the $N$-body Schrödinger equation under Bose symmetry. The system Hamiltonian describes pairwise particle interactions in the absence of an external potential. We derive a priori dispersive estimates that express the overall repulsive nature of the particle interactions. These estimates hold for a wide class of two-body interaction potentials which are independent of the particle number, $N$. We discuss applications of these estimates to the BBGKY hierarchy for reduced density matrices analyzed by Elgart, Erdös, Schlein and Yau.
\end{abstract}

Keywords: Dispersive estimates; interaction Morawetz type (correlation) estimates; many-body Hamiltonian; many-body Schrödinger equation; quantum hydrodynamics; BBGKY hierarchy.

Mathematics Subject Classification 2000: 35B45, 35Q40, 76Y05, 81V70, 82C22

\section{Introduction}

Quantum mechanics dictates that the dynamics of $N$ interacting particles at zero temperature be described by a wave function satisfying the time-dependent $N$-body Schrödinger equation. When $N$ is large this description becomes impractical. A common remedy is to reduce the system degrees of freedom by replacing the many-body Schrödinger equation by effective partial differential equations for 
single-particle wave functions. Thus, the many-body interaction is approximately replaced by sums of effective one-particle potentials. This approach amounts to a "mean-field" approximation, which is expected to be exact as $N \rightarrow \infty$ in an appropriate sense. This formulation yields nonlinear evolution laws in $3+1$ dimensions which often lead to successful predictions. In particular, the dynamics of particles with integer spin (Bosons) has been described by nonlinear Schrödinger-type equations $[12,13,25,30,31]$.

The relation of mean-field approaches to the many-particle Hamiltonian evolution is an area of active research. In settings with Boson symmetry, fundamental questions concern the rigorous derivation of nonlinear Schrödinger-type equations from $N$-body evolution laws and the formulation of theories that transcend mean field [3]. Recently, Elgart, Erdős, Schlein and Yau (EESY) [7-10] derived rigorously mean-field limits for Bosons on the basis of BBKGY-type hierarchies for reduced density matrices $[26,27]$.

In this paper, we study the $N$-particle Hamiltonian evolution of a Boson system for large yet finite $N$. We derive certain a priori estimates for hydrodynamic quantities, and examine some applications of these estimates to BBGKY hierarchies formulated for dilute atomic gases [7-10]. Our goal is to understand how many-body properties of the Boson system are connected to the mean-field limit.

The model equation for the particle system reads

$$
-i \partial_{t} \psi_{N}=H_{N} \psi_{N}
$$

where $\psi_{N}$ is the $N$-body wave function and $H_{N}$ is the Hamiltonian operator. This $H_{N}$ has the form

$$
H_{N}=\sum_{a} H_{a}+\sum_{a \neq b} H_{a b}, \quad a, b=1, \ldots, N,
$$

where $a$ and $b$ label the particles, $H_{a}$ is the part of the Hamiltonian acting solely on particle $a$, and $H_{a b}$ denotes the (pairwise) interaction between the particles $a$ and $b$. For identical particles, $H_{a}$ is described by

$$
H_{a}=-\Delta_{a}+V_{\text {trap }}\left(\vec{x}_{a}\right)
$$

and the interaction $H_{a b}$ has the form

$$
H_{a b}:=\frac{1}{2} V_{\mathrm{int}}\left(\vec{x}_{a}-\vec{x}_{b}\right),
$$

where $\vec{x}_{a}$ denotes the vector position of particle $a\left(\vec{x}_{a} \in \mathbb{R}^{3}\right), V_{\text {trap }}$ is a trapping potential which confines the particles in space, and $V_{\text {int }}$ is a two-body potential.

In our formulation, (1.1) is converted exactly to a set of hydrodynamic equations. A priori estimates for finite $N$ are derived here directly from these hydrodynamic equations. Note that by (1.1), we have $\psi_{N}(t)=U(t) \psi_{N}(0)$ where $U(t)=e^{i t H_{N}}$, a unitary operator. For large $N$, this representation of $\psi_{N}(t)$ is not particularly useful for a priori estimates and testable predictions. 
We proceed to discuss elements of the model. The trapping potential, $V_{\text {trap }}$, is introduced in experiments in order to keep the particles together. To avoid unecessary complications such as the effect of bound states in our analysis, we set $V_{\text {trap }}$ equal to zero, $V_{\text {trap }} \equiv 0$. The case with a nonzero external potential, $V_{\text {trap }} \not \equiv 0$, is the subject of future work.

Next, we comment on the particle interactions, which are crucial for the system evolution. In our model, the pairwise interaction, $V_{\text {int }}$, is considered as a known short-range potential. For dilute gases, which are the primary focus of this investigation, the particle collisions are sufficiently rare and weak; hence, the distance $\left|\vec{x}_{a}-\vec{x}_{b}\right|(a \neq b)$ remains mostly large in an appropriate sense. Consequently, each particle is influenced only by gross features of $V_{\text {int }}$. The specific details of this potential should be immaterial for our purposes. In this vein, by considering particles that repel each other, it suffices to assume that

$$
H_{a b}=V\left(\left|\vec{x}_{a}-\vec{x}_{b}\right|\right) ; \quad V \geq-c, V^{\prime}(s) \leq 0 \quad \forall s \in \mathbb{R}^{+} .
$$

Note that we consider only two-body interactions, neglecting effects of three-body and higher-order collisions [29].

A key ingredient of the particle model is the scattering length $l$ of the interaction potential, $V_{\text {int }}$. This $l$ encapsulates the gross features of the two-particle scattering [8]. Let the zero-energy scattering solution for the class of potentials $V_{\text {int }}(\vec{x})$ that decay sufficiently fast at infinity have the form $1-w_{0}(\vec{x})$ where $\lim _{|\vec{x}| \rightarrow \infty} w_{0}=0$; then, $l$ is defined by $l:=\lim _{|\vec{x}| \rightarrow \infty}\left(|\vec{x}| w_{0}\right)$. Accordingly, $V_{\text {int }}$ can be expressed in the scaled form $[8,21,22]$

$$
V_{\text {int }}(\vec{x})=l^{-2} V_{1}(|\vec{x}| / l),
$$

where $V_{1}$ is a fixed potential with scattering length equal to unity. Thus, the Hamiltonian $H_{N}$ has two parameters, namely, the number, $N$, of particles and the scattering length, $l$. In the EESY formulation [7-10], it is essential to allow for $N \rightarrow \infty$ by keeping $N l$ fixed. A parameter that enters the mean-field limit of (1.1) is [8]

$$
g:=4 \pi N l \text {. }
$$

Thus, setting $g=O(1)$ implies that the scattering length $l$ scales with $N^{-1}$.

The recent work by EESY [7-10] deserves special attention, because it provides a rigorous justification of the mean-field limit of (1.1) for Bosons via an averaging procedure. In Sec. 4, we outline their approach and derive certain a priori estimates. The uniqueness of the solution to the BBGKY hierarchy in the mean-field limit is also proved via a different argument by Klainerman and Machedon [16].

A crucial observation within the EESY framework is that, in order to be able to pass rigorously to the limit as $N$ increases, the two-body potential $V_{\text {int }}$ has to be appropriately scaled with $N$. For example, EESY [7-10] posit that $V_{\text {int }}$ approaches a delta function as $N \rightarrow \infty$, which comes from (1.6) with $l=N^{-1}$. These interactions cannot be treated as a perturbation since they have an appreciable effect for sufficiently large $N$. We note in passing that in a more elaborate model $V_{\text {int }}$ is 
replaced by the Fermi pseudopotential [2,14,15,17,18,29], an operator that consists of a delta function times a space derivative acting on $\psi_{N}$. The use of this pseudopotential allows for interesting extensions but causes mathematical difficulties and lies beyond our present scope.

A few remarks on a mean-field limit of (1.1) and its variants are in order. This limit can be derived heuristically under (1.6) with $l=O\left(N^{-1}\right)$ by approximately replacing the wave function $\psi_{N}$ by the tensor product

$$
\psi_{N} \approx \prod_{a=1}^{N} \phi_{a},
$$

where $\phi_{a}:=\phi\left(t, \vec{x}_{a}\right)$ and $\int \mathrm{d} \vec{x}|\phi|^{2}=1$. By manipulation of (1.1) and neglect of terms inconsistent with (1.8), a cubic nonlinear Schrödinger equation is recovered for $\phi(t, \vec{x})$. This equation reads $[11]$

$$
-i \partial_{t} \phi=H \phi+g|\phi|^{2} \phi-\mu(t) \phi
$$

where $H=-\Delta+V_{\text {trap }}$ and $\mu(t)=(g / 2) \int \mathrm{d} \vec{x}|\phi|^{4}$; cf. [12,13,25,30,31]. This form of $\mu$ does not appear in the results of Gross [12,13], Pitaevskii [25] and EESY [7-10] but is in agreement with the derivation by $\mathrm{Wu}[30,31]$. This $\mu(t)$ is not essential since it can be absorbed into a phase factor for $\phi$.

It is worthwhile mentioning the complementary view on particle dynamics that invokes operator-valued distributions [1,30,31]. For the periodic case, see, e.g. $[14,17,18]$. In this context, one defines the Boson annihilation operator $\Psi(\vec{x})$ and its adjoint, $\Psi^{*}(\vec{x})$, for a particle at point $\vec{x}$. Accordingly, $H_{N}$ with the Fermi pseudopotential is written in terms of $\Psi$ and $\Psi^{*}$. The annihilation operator $a_{0}(t)$ for the Bose condensate, the macroscopic quantum state in Bose-Einstein condensation, is introduced as the average $a_{0}(t)=\int \mathrm{d} \vec{x}\left\{\phi^{*} \Psi\right\}$, where $\phi(t, x)$ is the (oneparticle) condensate wave function. The evolution equation for $\phi$ can be derived by linearization of $H_{N}$ in $\Psi-\phi a_{0}$, which is considered as small in some sense, and enforcement of (1.1) under (1.8) [24,30,31]. This approach offers physical insight and is amenable to extensions, particularly the inclusion of higher-order scattering processes $[3,17,18,29,30]$. However, this formalism is less amenable to rigorous treatment. In particular, the introduction of the Fermi pseudopotential, which is a type of singular distribution, renders the analysis especially difficult.

Of course, tensor products such as (1.8) are not approximate solutions in any classical sense. So, an issue is how to make this type of approximation meaningful. The BBGKY hierarchy invoked by EESY [7-10], for which the Gross-Pitaevskii equation provides a closure condition, offers a meaningful scheme for justifying (1.8): if the Gross-Pitaevskii equation is satisfied then all the equations in the hierarchy are satisfied as $N \rightarrow \infty$.

To understand the system evolution by connecting macroscopic variables such as $\phi$ with microscopic quantities for finite $N$, we resort to a priori dispersive estimates which express the particle repulsions. These estimates are scale independent: we derive them for interaction potentials of the form $V\left(\left|\vec{x}_{a}-\vec{x}_{b}\right|\right)$, omitting the 
scaling which can be inserted in the end. Our basic underlying assumption is given by $(1.5)$.

Our a priori estimates are useful for the passage to the limit $N \rightarrow \infty$, but also offer insight into the case with finite $N$. The techniques employed in the present work originated in $[4,23]$ in connection to the nonlinear Schrödinger equation (see also $[5,28])$. A new element of the present problem is the presence of a large number of interaction potentials and the fact that the hydrodynamic momenta are not conserved by the flow. Our analysis addresses the question in what sense the potentials are repulsive and what are the precise implications of this repulsive nature.

The static version of (1.1) is not addressed here. This case was studied by Dyson [6] and by Lee, Huang and Yang [17]. A mathematical proof of the BoseEinstein condensation for the time-independent case was provided recently by Lieb et al. [19-22].

The remainder of the paper is organized as follows. In Sec. 2, we derive an a priori space-time estimate for the particle density function after the collapse (identification) of two particle positions. Boson symmetry is not required for the main result of Sec. 2. In Sec. 3, we restrict attention to Bosons and derive another estimate for the square of an appropriately averaged (reduced) particle density. In Sec. 4, we outline the EESY approach based on the BGGKY hierarchy for reduced density matrices [7-10,16], and derive a priori estimates in the context of this formulation. The (Einstein) summation convention for repeated indices is employed unless it is noted otherwise.

\section{First Estimate by Coordinate Collapse}

In this section, we derive an a priori space-time estimate for the particle density of the $N$-body system described by (1.1)-(1.4) when $N$ is large and finite. Boson symmetry is not required for our main result but simplifies it considerably, as shown below. Our motivation is to investigate in what sense the wave function $\psi_{N}$ disperses with time. The methodology followed here originates from the work of Lin and Strauss [23].

Specifically, we show that

$$
\sum_{a \neq b} \int \mathrm{d} t \mathrm{~d} \vec{X}_{a b} \rho\left(t, \vec{X}_{a b}\right) \leq N^{2}\left\|\psi_{N}\right\|_{H^{1}}\left\|\psi_{N}\right\|_{L^{2}},
$$

where the particle density function $\rho$ is defined by

$$
\rho\left(t, \vec{x}_{1}, \ldots \vec{x}_{N}\right):=\frac{1}{2}\left|\psi_{N}\left(t, \vec{x}_{1}, \ldots \vec{x}_{N}\right)\right|^{2} .
$$

The reduced vector $\vec{X}_{a b}$ entering (2.1) comes from a basic collapse mechanism which identifies two of the variables $\left(\vec{x}_{1}, \ldots, \vec{x}_{N}\right)$. Hence, $\vec{X}_{a b}$ is defined by

$$
\vec{X}_{a b}:=(\vec{X})_{\vec{x}_{a}=\vec{x}_{b}} \in \mathbb{R}^{3(N-1)}, \quad \vec{X}:=\left(\vec{x}_{1}, \vec{x}_{2}, \ldots, \vec{x}_{N}\right) .
$$


See Sec. 4 for a discussion on the motivation for this coordinate collapse. For Boson particles, $\psi_{N}$ remains invariant under permutations of $\vec{x}_{a}$ 's. Thus, the integrals entering the sum of (2.1) are the same and the estimate (2.1) evidently becomes

$$
m_{a b}:=\int \mathrm{d} t \mathrm{~d} \vec{X}_{a b}\left\{\rho\left(t, \vec{X}_{a b}\right)\right\} \leq\left\|\psi_{N}\right\|_{H^{1}}\left\|\psi_{N}\right\|_{L^{2}}
$$

Estimates (2.1) and (2.4) can be modified to manifestly become independent of the number, $N$, of particles. As a matter of fact, these estimates are not optimal since the term $\left\|\psi_{N}\right\|_{H^{1}}$ scales as $N^{1 / 2}$. We show that (2.1) can be replaced by

$$
\sum_{a \neq b} \int \mathrm{d} t \mathrm{~d} \vec{X}_{a b} \rho\left(t, \vec{X}_{a b}\right) \leq N^{3 / 2}\left\|\vec{\nabla} \psi_{N}\right\|_{L^{2}}\left\|\psi_{N}\right\|_{L^{2}},
$$

where $\vec{\nabla}=\left(\vec{\nabla}_{1}, \vec{\nabla}_{2}, \ldots, \vec{\nabla}_{N}\right)$. In the same vein, estimate (2.4) (for Bosons) is modified to

$$
m_{a b} \leq N^{-1 / 2}\left\|\nabla \psi_{N}\right\|_{L^{2}}\left\|\psi_{N}\right\|_{L^{2}}
$$

The independence from $N$ of these modified estimates becomes explicit for initial data that form a tensor product, as discussed at the end of this section.

We proceed to prove (2.1). Our program consists of the following steps. (i) An evolution equation is derived for the particle momentum density, expressing the fact that momentum is not conserved by the flow (see (2.14)). (ii) This equation is contracted with suitable vector fields to yield (2.19), which contains "error" terms depending on the nature of particle interactions. (iii) Estimates for these error terms are obtained directly. (iv) The contracted equation is integrated in space and time to yield (2.1).

We now describe the procedure in detail. The evolution equation (1.1) is recast to the form

$$
i \partial_{t} \psi_{N}+\left(\sum_{a} H_{a}\right) \psi_{N}+\left(\sum_{a \neq b} H_{a b}\right) \psi_{N}=0,
$$

where $H_{a}=-\Delta_{a}$ and $H_{a b}=V\left(\left|\vec{x}_{a}-\vec{x}_{b}\right|\right)$, which implies that our analysis will be independent of any particular scaling of the interaction potential. By (1.5), the basic assumption is

$$
V^{\prime}(s) \leq 0 \quad \forall s \in \mathbb{R}^{+}
$$

The particle coordinates are denoted by $x_{a}^{j}$ where $j=1,2,3$ and $a=1,2, \ldots, N$. We consider the Euclidean space with metric

$$
g_{j k}^{a b}:=\delta_{j k} \delta^{a b}, \quad j, k=1,2,3, \quad a, b=1,2, \ldots, N,
$$

and use the notation

$$
\nabla_{a}^{j}:=\frac{\partial}{\partial x_{a}^{j}}
$$


Next, we define the momentum variables and stress tensor. The components of the momentum density for the ath particle are defined by

$$
p_{a}^{j}:=\frac{1}{2 i}\left(\psi_{N}^{*} \nabla_{a}^{j} \psi_{N}-\psi_{N} \nabla_{a}^{j} \psi_{N}^{*}\right), \quad j=1,2,3, \quad a=1,2 \ldots N,
$$

where $\psi_{N}^{*}$ is the complex conjugate of $\psi_{N}$. The stress tensor is

$$
\sigma_{a b}^{j k}:=\left(\nabla_{a}^{j} \psi_{N}\right)\left(\nabla_{b}^{k} \psi_{N}^{*}\right)+\left(\nabla_{a}^{j} \psi_{N}^{*}\right)\left(\nabla_{b}^{k} \psi_{N}\right), \quad j, k=1,2,3, \quad a, b=1, \ldots, N .
$$

For completeness, we provide the conservation law for the density $\rho$, although this law is not invoked directly in the proof. The mass conservation statement reads

$$
\partial_{t}\{\rho\}-\nabla_{j}^{a}\left\{p_{a}^{j}\right\}=0 .
$$

Use of this law will be made at the end of this section and in Sec. 3.

The evolution equation for the momenta $p_{j}^{a}$ stems from differentiation of (2.11) with respect to time and use of (1.1). The resulting equation reads

$$
\partial_{t}\left\{p_{a}^{j}\right\}-\nabla_{b}^{k}\left\{\sigma_{k a}^{j b}\right\}+\nabla_{a}^{j}\{\boldsymbol{\Delta} \rho\}-\sum_{b, b \neq a} 2 V^{\prime}\left(\left|\vec{x}_{a}-\vec{x}_{b}\right|\right) \frac{x_{a}^{j}-x_{b}^{j}}{\left|\vec{x}_{a}-\vec{x}_{b}\right|} \rho=0,
$$

where $\boldsymbol{\Delta}:=\sum_{a} \Delta_{a}$ is the $3 N$-dimensional Laplacian. Equation (2.14) is not a conservation law because of $V^{\prime}$ on the left-hand side. By defining the weights $w_{a b}$ and the $3 N$-dimensional vector $\vec{M}_{a}=\left(\vec{M}^{1}, \vec{M}^{2}, \ldots, \vec{M}^{N}\right)$ according to

$$
w_{a b}:=-2 V^{\prime}\left(\left|\vec{x}_{a}-\vec{x}_{b}\right|\right), \quad \vec{M}_{a}:=\sum_{b, b \neq a} w_{a b} \frac{\vec{x}_{a}-\vec{x}_{b}}{\left|\vec{x}_{a}-\vec{x}_{b}\right|},
$$

we rewrite $(2.14)$ as

$$
\partial_{t}\left\{\vec{p}^{a}\right\}-\nabla_{k}^{b}\left\{\vec{\sigma}_{b}^{k a}\right\}+\vec{\nabla}^{a}\{\boldsymbol{\Delta} \rho\}+\vec{M}^{a} \rho=0, \quad a=1,2, \ldots, N .
$$

For algebraic convenience we have written $\vec{p}^{a}:=\left(p_{1}^{a}, p_{2}^{a}, p_{3}^{a}\right)$, the three-vector with respect to the group of coordinates for $\vec{x}_{a}$. Notice that the condition $V^{\prime} \leq 0$ for repulsive interactions entails $w_{a b}=w_{b a} \geq 0$.

Next, we contract the momentum equation, (2.14) or (2.16), with a suitable $3 N$ dimensional vector field, $\vec{Y}_{(a b)}$. This field consists of $N$ ordered three-vectors and is defined by

$$
\vec{Y}_{(a b)}:=\left(\overrightarrow{0}, \ldots, \overrightarrow{0}, \frac{\vec{x}_{a}-\vec{x}_{b}}{\left|\vec{x}_{a}-\vec{x}_{b}\right|}, \overrightarrow{0}, \ldots, \overrightarrow{0}, \frac{\vec{x}_{b}-\vec{x}_{a}}{\left|\vec{x}_{b}-\vec{x}_{a}\right|}, \overrightarrow{0}, \ldots, \overrightarrow{0}\right) .
$$

This expression means that the sole nonzero three-vectors forming $Y_{(a b)}$ are the ones in the $a$ th and $b$ th position in the way indicated above. We adopt the convention that $\vec{Y}_{(a b)}=\vec{Y}_{(b a)}$ and write $Y_{c(a b)}^{j}$ to denote the $j$ th component of the vector located 
in the $c$ th position of $\vec{Y}_{(a b)}(j=1,2,3$ and $a, b, c=1, \ldots, N)$. The contraction of the ath-particle momentum, $\vec{p}^{a}$, with $Y_{a(c d)}$ equals

$$
p_{j}^{a} Y_{a(c d)}^{j}=\vec{p}_{c} \cdot \frac{\vec{x}_{c}-\vec{x}_{d}}{\left|\vec{x}_{c}-\vec{x}_{d}\right|}+\vec{p}_{d} \cdot \frac{\vec{x}_{d}-\vec{x}_{c}}{\left|\vec{x}_{d}-\vec{x}_{c}\right|},
$$

where no summation over $c$ and $d$ is implied. The contraction of (2.14) with the field $Y_{a(c d)}^{j}$ by (2.17) produces the equation

$$
\begin{aligned}
& \partial_{t}\left\{p_{j}^{a} Y_{a(c d)}^{j}\right\}-\nabla_{k}^{b}\left\{\sigma_{j b}^{k a} Y_{a(c d)}^{j}\right\}+\nabla_{j}^{a}\left\{Y_{a(c d)}^{j} \Delta \rho\right\} \\
& \quad+\left(\nabla_{k}^{b} Y_{a(c d)}^{j}\right) \sigma_{j b}^{k a}-\left(\nabla_{j}^{a} Y_{a(c d)}^{j}\right) \Delta \rho+\sum_{c} w_{a c} \frac{x_{j}^{a}-x_{j, c}}{\left|\vec{x}_{a}-\vec{x}_{c}\right|} Y_{a(c d)}^{j} \rho=0 .
\end{aligned}
$$

We will show that the dispersive nature of (2.19) provides an a priori bound for the collapsed density according to (2.1). For this purpose, we integrate (2.19) over the time-slice $[0, T] \times \mathbb{R}^{3 N}$. The last two terms in the first line of $(2.19)$ are explicitly integrated out to zero, viz.

$$
\int \mathrm{d} t \mathrm{~d} \vec{X} \nabla_{k}^{b}\left\{\sigma_{j b}^{k a} Y_{a(c d)}^{j}\right\}=0=\int \mathrm{d} t \mathrm{~d} \vec{X} \nabla_{j}^{a}\left\{Y_{a(c d)}^{j} \Delta \rho\right\} .
$$

We will return to the first term, $\partial_{t}\left\{p_{j}^{a} Y_{a(c d)}^{j}\right\}$, near the end of this proof.

Our main task now is to investigate the nature of terms in the second line of (2.19). We sum over all contractions with the vector fields $\vec{Y}_{(c d)}$ for $c \neq d$. There are $N(N-1) / 2$ different vector fields. For simplicity, we will relabel indices by $c=a, d=b$ and $a=c$.

First, we focus on the term $-\left(\nabla_{j}^{a} Y_{a(c d)}^{j}\right) \Delta \rho$, which is integrated to furnish

$$
\sum_{c \neq d} \int \mathrm{d} t \mathrm{~d} \vec{X}\left\{-\left(\nabla_{j}^{a} Y_{a(c d)}^{j}\right) \Delta \rho\right\}, \quad \vec{X}=\left(\vec{x}_{1}, \vec{x}_{2}, \ldots, \vec{x}_{N}\right) .
$$

The identity

$$
\operatorname{div} \vec{Y}_{(a b)}=\vec{\nabla} \cdot \vec{Y}_{(a b)}=\frac{4}{\left|\vec{x}_{a}-\vec{x}_{b}\right|}
$$

and the subsequent integration by parts in (2.21) with $c=a$ and $d=b$ yield

$$
\sum_{a \neq b} \int \mathrm{d} t \mathrm{~d} \vec{X}\left\{\frac{-4}{\left|\vec{x}_{a}-\vec{x}_{b}\right|}\right\} \Delta \rho=c \sum_{a \neq b} \int \mathrm{d} t \mathrm{~d} \vec{X}_{a b}\left\{\rho\left(t, \vec{X}_{a b}\right)\right\} \geq 0
$$

where $\boldsymbol{\Delta}_{a}\left(\left|\vec{x}_{a}-\vec{x}_{b}\right|^{-1}\right)=4 \pi \delta\left(\vec{x}_{a}-\vec{x}_{b}\right)$ and $\vec{X}_{a b}$ is defined in (2.3). Notice that in our derivation so far, we did not have to assume that the particles are Bosons. However, it should be borne in mind that in the case with Bosons the results are simplified since all these integrals are equal. 
Next, we focus on the last term in (2.19), viz.

$$
\mathcal{E}:=\sum_{a \neq b}\left(\vec{M}^{a} \cdot \frac{\vec{x}_{a}-\vec{x}_{b}}{\left|\vec{x}_{a}-\vec{x}_{b}\right|}+\vec{M}^{b} \cdot \frac{\vec{x}_{b}-\vec{x}_{a}}{\left|\vec{x}_{b}-\vec{x}_{a}\right|}\right) \rho(t, \vec{X}),
$$

which by use of (2.15) can be written explicitly as the triple sum

$$
\sum_{a \neq b} \sum_{c}\left(w_{c a} \frac{\vec{x}_{c}-\vec{x}_{a}}{\left|\vec{x}_{c}-\vec{x}_{a}\right|} \cdot \frac{\vec{x}_{b}-\vec{x}_{a}}{\left|\vec{x}_{b}-\vec{x}_{a}\right|}+w_{c b} \frac{\vec{x}_{c}-\vec{x}_{b}}{\left|\vec{x}_{c}-\vec{x}_{b}\right|} \cdot \frac{\vec{x}_{a}-\vec{x}_{b}}{\left|\vec{x}_{a}-\vec{x}_{b}\right|}\right) \rho(t, \vec{X}) .
$$

Terms with $c=b$ or $c=a$ in the first or second sum of (2.25), respectively, add up to a manifestly positive term. We turn our attention to terms with $c \neq a, b$. Let us pair the term

$$
w_{c a} \frac{\vec{x}_{c}-\vec{x}_{a}}{\left|\vec{x}_{c}-\vec{x}_{a}\right|} \cdot \frac{\vec{x}_{b}-\vec{x}_{a}}{\left|\vec{x}_{b}-\vec{x}_{a}\right|},
$$

which comes from the contraction with $Y_{(a b)}$, with the term

$$
w_{a c} \frac{\vec{x}_{a}-\vec{x}_{c}}{\left|\vec{x}_{a}-\vec{x}_{c}\right|} \cdot \frac{\vec{x}_{b}-\vec{x}_{c}}{\left|\vec{x}_{b}-\vec{x}_{c}\right|},
$$

which comes from the contraction with $Y_{(b c)}$. The sum of these two terms admits a geometric interpretation as follows. Consider the triangle $T\left(\vec{x}_{a}, \vec{x}_{b}, \vec{x}_{c}\right)$ with vertices $\vec{x}_{a}, \vec{x}_{b}$ and $\vec{x}_{c}$ and denote the corresponding angles $\alpha_{a}:=\alpha(a), \alpha_{b}:=\alpha(b)$ and $\alpha_{c}:=\alpha(c)$ as shown in Fig. 1. Evidently, the sum of terms (2.26) and (2.27) equals

$$
w_{a c}\left[\cos \left(\alpha_{a}\right)+\cos \left(\alpha_{c}\right)\right] \geq 0,
$$

which has a positive sign. In a similar manner, one can pair the term

$$
w_{c b} \frac{\vec{x}_{c}-\vec{x}_{b}}{\left|\vec{x}_{c}-\vec{x}_{b}\right|} \cdot \frac{\vec{x}_{a}-\vec{x}_{b}}{\left|\vec{x}_{a}-\vec{x}_{b}\right|}
$$

which comes from the contraction with $Y_{(a b)}$, with the term

$$
w_{b c} \frac{\vec{x}_{b}-\vec{x}_{c}}{\left|\vec{x}_{b}-\vec{x}_{c}\right|} \cdot \frac{\vec{x}_{a}-\vec{x}_{c}}{\left|\vec{x}_{a}-\vec{x}_{c}\right|}
$$

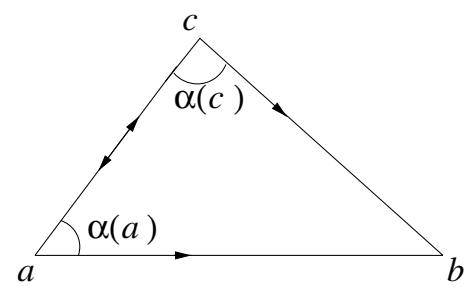

Fig. 1. Geometric interpretation of interaction terms from (2.26) and (2.27) by the triangle $T\left(\vec{x}_{a}, \vec{x}_{b}, \vec{x}_{c}\right)$. 
which comes from the contraction with $Y_{(a c)}$. The representation by the triangle of Fig. 1 implies that the sum of the last two terms is

$$
w_{c b}\left[\cos \left(\alpha_{b}\right)+\cos \left(\alpha_{c}\right)\right] \geq 0 .
$$

As a matter of fact, by inspection of the sum of (2.26), and (2.27), we can define the potential

$$
U\left(\vec{x}_{a}, \vec{x}_{b}, \vec{x}_{c}\right):=w_{c a} \frac{\vec{x}_{c}-\vec{x}_{a}}{\left|\vec{x}_{c}-\vec{x}_{a}\right|} \cdot \frac{\vec{x}_{b}-\vec{x}_{a}}{\left|\vec{x}_{b}-\vec{x}_{a}\right|}+w_{a c} \frac{\vec{x}_{a}-\vec{x}_{c}}{\left|\vec{x}_{a}-\vec{x}_{c}\right|} \cdot \frac{\vec{x}_{b}-\vec{x}_{c}}{\left|\vec{x}_{b}-\vec{x}_{c}\right|} .
$$

Accordingly, the quantity $\mathcal{E}$ from (2.24) or (2.25) is recast to the expression

$$
\mathcal{E}=\sum_{a \neq b} \sum_{c} U\left(\vec{x}_{a}, \vec{x}_{b}, \vec{x}_{c}\right) \rho(t, \vec{X}) .
$$

There are three remarks in order on expression (2.33).

Remark 2.1. There are overall $N(N-1)^{2}$ terms in the summation (2.33), because each contraction produces $2(N-1)$ terms and there are $N(N-1) / 2$ contractions. The number of terms with $c=b$ or $c=a$, which have positive sign, is $N(N-1)$; thus, the number of the remaining terms is $N(N-1)(N-2)$ and we separate them into two groups.

Remark 2.2. Notice the following term, which comes from (2.26):

$$
-V^{\prime}\left(\left|\vec{x}_{c}-\vec{x}_{a}\right|\right) \frac{\vec{x}_{c}-\vec{x}_{a}}{\left|\vec{x}_{c}-\vec{x}_{a}\right|} \cdot \frac{\vec{x}_{b}-\vec{x}_{a}}{\left|\vec{x}_{b}-\vec{x}_{a}\right|} \rho(t, \vec{X}) .
$$

This term can be thought of as a three-particle interaction: the particle $c$ interacts with $a$ and $b$. If we consider the plane $P_{a, b}$ that passes through $\vec{x}_{a}$ and is orthogonal to the vector $\vec{x}_{b}-\vec{x}_{a}$, then in the half space that contains $\vec{x}_{b}$ the expression (2.34) is positive. In contrast, in the complementary half space this expression becomes negative. The idea is that (2.34) can be paired with another term where the particle $a$ interacts with $b$ and $c$ so that the sum of the two contributions is positive. This argument gives general qualitative information on the particle mutual repulsion.

Remark 2.3. By replacing the vector fields $\vec{X}$ in $(2.17)$ with $\left(\vec{x}_{a}-\vec{x}_{b}-\vec{d}\right) / \mid \vec{x}_{a}-$ $\vec{x}_{b}-\vec{d} \mid$, where $\vec{d}$ is some fixed vector, we obtain an estimate like the one in (2.4), where the integration is over $\vec{x}_{a}-\vec{x}_{b}=\vec{d}$.

These remarks conclude the investigation of the last term in evolution law (2.19). We have asserted that this term is positive.

The first term in the second line of (2.19) reads

$$
\Sigma:=\left(\nabla_{k}^{b} Y_{a(c d)}^{j}\right) \sigma_{j b}^{k a} .
$$

Evidently, this term is positive, i.e. $\Sigma \geq 0$ for the given vector fields.

It remains to collect the results obtained thus far in order to derive (2.4). In summary, with regard to the second line of (2.19), we showed that the first and third terms are positive and the second term yields the positive integral (2.23). 
Recall that (2.20) hold. By integrating the first term in (2.19) over the time-slice $[0, T] \times \mathbb{R}^{3 N}$, we obtain the estimate

$$
\begin{aligned}
0 \leq \int_{0}^{T} \mathrm{~d} t \int \mathrm{d} \vec{X}\left\{-\partial_{t}\left(p_{j}^{a} Y_{a(c d)}^{j}\right)\right\} & =\int \mathrm{d} \vec{X}\left[\left.\left(p_{j}^{a} Y_{a(c d)}^{j}\right)\right|_{t=0}-\left.\left(p_{j}^{a} Y_{a(c d)}^{j}\right)\right|_{T}\right] \\
& \leq\left\|\psi_{N}\right\|_{L^{2}}\left\|\psi_{N}\right\|_{H^{1}} .
\end{aligned}
$$

Here, we used definition (2.11) for the momentum variables. This statement concludes the proof of estimate (2.1).

We proceed to show (2.5). First, we observe that (2.1) is recast to the form

$$
\sum_{a \neq b} m_{a b} \leq \sup _{t}\left|\int d \vec{X}\{\vec{Y} \cdot \vec{P}\}\right|
$$

where $\vec{P}$ is the momentum, $\vec{Y}$ is a vector field defined by $\vec{Y}=\sum_{c \neq d} \vec{Y}_{(c d)}$ and each $\vec{Y}_{(c d)}$ here has unit length; see (2.17). Therefore, if we write $\vec{Y}=\left(\vec{Y}_{1}, \vec{Y}_{2}, \ldots, \vec{Y}_{N}\right)$ then we conclude that $\left|\vec{Y}_{a}\right| \leq N$.

Next, let us recall that the momenta are given by $\vec{P}=\left(\vec{p}_{1}, \vec{p}_{2}, \ldots, \vec{p}_{N}\right)$ where each $\vec{p}_{a}$ is defined by (2.11). Therefore, Cauchy-Schwartz implies that

$$
\int \mathrm{d} \vec{X}\left\{\left|\vec{p}_{a}\right|\right\} \leq\left\|\psi_{N}\right\|_{L^{2}}\left\|\vec{\nabla}_{a} \psi_{N}\right\|_{L^{2}}
$$

Furthermore, we have the inequality

$$
|\vec{Y} \cdot \vec{P}| \leq \sum_{a}\left|\vec{Y}_{a} \cdot \vec{p}_{a}\right| \leq N \sum_{a}\left|\vec{p}_{a}\right|
$$

Thus, by integration and use of Cauchy-Schwartz as in (2.38), we find the bound

$$
\int \mathrm{d} \vec{X}\left\{\sum_{a}\left|\vec{p}_{a}\right|\right\} \leq \sum_{a}\left\|\psi_{N}\right\|\left\|\vec{\nabla}_{a} \psi_{N}\right\| \leq\left\|\psi_{N}\right\| N^{1 / 2}\left(\sum_{a}\left\|\vec{\nabla}_{a} \psi_{N}\right\|^{2}\right)^{1 / 2}
$$

where $\|\cdot\|$ here denotes the $L^{2}$ norm. The combination of (2.39) and (2.40) yields $(2.5)$.

Next we discuss implications of estimate (2.5) for initial $\psi_{N}$ that is a tensor product of one-particle states. First, note that the energy of the $N$-body system is defined by

$$
E_{N}(t):=\int_{\mathbb{R}^{3 N}} \mathrm{~d} \vec{X}\left\{\frac{1}{2} \operatorname{tr}(\sigma)+\sum_{a \neq b} V\left(\left|\vec{x}_{a}-\vec{x}_{b}\right|\right) 2 \rho\right\}
$$

This quantity is conserved, i.e. $\mathrm{d} E_{N} / \mathrm{d} t \equiv 0$. It is written in the familiar form

$$
E_{N}(t):=\int_{R^{3 N}} \mathrm{~d} \vec{X}\left\{\left|\vec{\nabla} \psi_{N}\right|^{2}+\sum_{a \neq b} V\left(\left|\vec{x}_{a}-\vec{x}_{b}\right|\right)\left|\psi_{N}\right|^{2}\right\} .
$$


If the initial data form a tensor product, $\psi_{N}(0, \vec{X})=\prod_{a=1}^{N} \phi\left(\vec{x}_{a}\right)$, then the total energy scales as $E_{N}(0)=N E_{1}$ where $E_{1}$ is the energy per particle. Then, we have the inequality $\left\|\vec{\nabla} \psi_{N}\right\|_{L^{2}} \leq N^{1 / 2} \sqrt{E_{1}}$. Therefore, by (2.5), we obtain the bound

$$
\sum_{a \neq b} m_{a b} \leq N^{2}\left\|\psi_{N}\right\|_{L^{2}} \sqrt{E_{1}} .
$$

In the case where all particles are Bosons, the last bound reduces to

$$
m_{a b} \leq \sqrt{E_{1}}\left\|\psi_{N}\right\|_{L^{2}}
$$

which is manifestly independent of $N$. This property is crucial if one wants to pass to the limit $N \rightarrow \infty$. (Notice that $\left\|\psi_{N}\right\|$ here is independent of $N$.)

We close this section with the derivation of another estimate expressing the dispersion of the particle density $\rho$. This estimate concerns the evolution of the variance of $\rho$, viz.

$$
\frac{\mathrm{d}^{2}}{\mathrm{~d} t^{2}} \int \mathrm{d} \vec{X}\{\rho D\} \geq 0,
$$

where the weight function $D(\vec{X})$ is defined by

$$
D\left(\vec{x}_{1}, \vec{x}_{2}, \ldots, \vec{x}_{N}\right):=\sum_{a \neq b}\left|\vec{x}_{a}-\vec{x}_{b}\right| .
$$

This $D$ is intimately connected with the procedure applied above. Indeed, the derivative of $D$ reads

$$
\vec{\nabla}^{a} D=\sum_{b \neq a} \frac{\vec{x}_{a}-\vec{x}_{b}}{\left|\vec{x}_{a}-\vec{x}_{b}\right|}
$$

With the introduction of $Y_{j}^{a}$ by

$$
\nabla_{j}^{a} D\left(\vec{x}_{1}, \vec{x}_{2} \ldots \vec{x}_{N}\right)=: Y_{j}^{a}
$$

it becomes evident that we actually performed contractions with the vector fields $Y_{j}^{a}$ for $a=1,2, \ldots, N$. Estimate (2.45) for $\rho$ results from the combination of $(2.46)$ with the mass conservation law (2.13).

A possible extension of our analysis is the inclusion of a nonzero trapping potential, $V_{\text {trap. }}$. To speculate the related difficulties, we recall that the repulsive nature of particle interactions means that the particles tend to disperse so there are no bound states. The addition of a trapping potential in the Hamiltonian will produce an effective attractive force that keeps the particles together. This influence will compete with the dispersive effect described above, creating the possibility for trapped states. 


\section{Second Estimate by Commutator Operators}

In this section, we restrict attention to Bosons and derive an a priori estimate that involves a space-time integral of the square of a reduced particle density function. We prove this estimate by resorting to vector commutator operators and the evolution of a suitably defined action and associated correlation function.

Specifically, we show that

$$
\sum_{a \neq b} \int_{\mathbb{R} \times \mathbb{R}^{3}} \mathrm{~d} t \mathrm{~d} \vec{x}_{a-b}\left\{\left(\widetilde{\rho}_{a, b}\left(t, \vec{x}_{a b}\right)\right)^{2}\right\} \leq N^{2}\left\|\psi_{N}\right\|_{H^{1}}\left\|\psi_{N}\right\|_{L^{2}}^{3},
$$

where the averaged, reduced density $\widetilde{\rho}_{a, b}$ is defined by

$$
\widetilde{\rho}_{a, b}\left(t, \vec{x}_{a-b}\right):=\int_{\mathbb{R}^{3(N-1)}} \mathrm{d} \vec{X}_{a, b} \mathrm{~d} \vec{x}_{a+b}\{\rho\} .
$$

In the above, the variables $\vec{x}_{a \pm b}$ denote the center-of-mass coordinates

$$
\vec{x}_{a \pm b}:=\frac{1}{\sqrt{2}}\left(\vec{x}_{a} \pm \vec{x}_{b}\right)
$$

and the reduced coordinates $\vec{X}_{a, b}$ are defined by

$$
\vec{X}_{a, b}:=\left(\vec{x}_{c}\right)_{c \neq a, b} \in \mathbb{R}^{3(N-2)},
$$

i.e. $\vec{X}_{a, b}$ stem from $\left(\vec{x}_{1}, \vec{x}_{2}, \ldots, \vec{x}_{N}\right)$ with the pair $\left(\vec{x}_{a}, \vec{x}_{b}\right)$ being omitted.

By analogy with Sec. 2, we also modify (3.1) to the estimate

$$
\sum_{a \neq b} \int_{\mathbb{R}^{\prime} \mathbb{R}^{3}} \mathrm{~d} t \mathrm{~d} \vec{x}_{a-b}\left\{\left(\widetilde{\rho}_{a, b}\left(t, \vec{x}_{a-b}\right)\right)^{2}\right\} \leq N^{3 / 2}\|\nabla \psi\|_{L^{2}}\|\psi\|_{L^{2}}^{3},
$$

which is manifestly independent of the number, $N$, of particles. The case with initial data that form a tensor product is discussed at the end of this section.

First, we briefly review and comment on the main assumptions and starting equations. The many-particle wave function satisfies (2.7), which is recast to the equation

$$
i \partial_{t} \psi_{N}-\Delta \psi_{N}+\sum_{a \neq b} V\left(\left|\vec{x}_{a}-\vec{x}_{b}\right|\right) \psi_{N}=0,
$$

where $\boldsymbol{\Delta}$ is the Laplacian in $\mathbb{R}^{3 N}$. The key idea in this section is to invoke commutator vector operators, which we apply to conservation laws stemming from (3.6). In addition to condition (2.8) on $V$, we impose Bose symmetry, i.e. require that $\psi_{N}$ remain invariant under permutation of any coordinate pair $\left(\vec{x}_{a}, \vec{x}_{b}\right)$ :

$$
\psi_{N}\left(t, \ldots, \vec{x}_{a}, \ldots, \vec{x}_{b}, \ldots\right)=\psi_{N}\left(t, \ldots, \vec{x}_{b}, \ldots, \vec{x}_{a}, \ldots\right) .
$$

This property is inherited by the density function defined by (2.2), viz.

$$
\rho\left(t, \ldots, \vec{x}_{a}, \ldots, \vec{x}_{b}, \ldots\right)=\rho\left(t, \ldots, \vec{x}_{b}, \ldots, \vec{x}_{a}, \ldots\right) .
$$


Crucial in our considerations are the evolution laws (2.13) and (2.14) for mass density and momentum. These equations read

$$
\begin{aligned}
\partial_{t} \rho-\nabla_{k}^{b} p_{b}^{k} & =0, \\
\partial_{t} p_{j}^{a}-\nabla_{k}^{b}\left\{\sigma_{j b}^{k a}+\delta_{j}^{k} \delta_{b}^{a}(-\Delta \rho)\right\}+M_{j}^{a} \rho & =0,
\end{aligned}
$$

where $j=1,2,3$ and $a=1,2, \ldots, N$. The relevant quantities, namely, the density $\rho$, the momenta $p_{j}^{a}$, and the stress tensor $\sigma_{j k}^{a b}$ are defined by $(2.2),(2.11)$ and (2.12). In particular, $\sigma_{j k}^{a b}$ is rewritten as

$$
\sigma_{j k}^{a b}=\rho^{-1}\left[p_{j}^{a} p_{k}^{b}+\left(\nabla_{j}^{a} \rho\right)\left(\nabla_{k}^{b} \rho\right)\right] .
$$

It is worthwhile recalling the conservation of the total energy, $E_{N}(t)$, which we will not use directly here. This law can be expressed as the conservation of the integral (2.41): this quantity is a constant $(\mathrm{d} E / \mathrm{d} t \equiv 0)$ and controls the right-hand side of (3.1) provided that $V$ is bounded below (see (1.5)).

The proof of (3.1) is based on the construction of suitable vector commutator operators and study of associated evolution laws. Our program consists of the following steps. (i) A vector commutator operator, $\vec{C}$, is constructed as a suitable average over all particles. (ii) An action, $L(t)$, is defined as the inner product $\langle\vec{C} \cdot \vec{P}(t) \mid \rho(t)\rangle$ where $\vec{P}$ is the $3 N$-dimensional momentum vector. (iii) The evolution of $L(t)$ is described in terms of distinct inner products, $S_{\ell}$. (iv) Estimates are derived for each $S_{\ell}$. (v) The evolution equation for $L(t)$ is integrated to yield (3.1).

We proceed to carry out this program. For each particle pair $(a, b)$, we construct the integral operator $B_{a b}$ by

$$
\left(B_{a b} f\right)\left(\vec{x}_{a-b}\right):=\int_{\mathbb{R}^{3 N}} \frac{\mathbf{1}\left(\vec{x}_{a+b}^{\prime}\right) \mathbf{1}\left(\vec{X}_{a, b}^{\prime}\right)}{\left|\vec{x}_{a-b}-\vec{x}_{a-b}^{\prime}\right|} f\left(\vec{X}^{\prime}\right) \mathrm{d} \vec{X}^{\prime} .
$$

By using these $B_{a b}$, we construct the $3 N$-vector commutator operator

$$
\vec{C}_{a ; b}:=\left(0 \ldots 0,\left[\vec{x}_{a-b} ; B_{a b}\right], 0 \ldots 0\right),
$$

where $[\mathcal{A} ; \mathcal{B}]:=\mathcal{A B}-\mathcal{B} \mathcal{A}$ denotes the commutator of $\mathcal{A}$ and $\mathcal{B}$, and $\left[\vec{x}_{a-b} ; B_{a b}\right]$ in (3.13) is placed in the ath position and acts on functions $f$ according to

$$
\left(\left[\vec{x}_{a-b} ; B_{a b}\right] f\right)\left(\vec{x}_{a-b}\right)=\int_{\mathbb{R}^{3 N}} \frac{\vec{x}_{a-b}-\vec{x}_{a-b}^{\prime}}{\left|\vec{x}_{a-b}-\vec{x}_{a-b}^{\prime}\right|} f\left(\vec{X}^{\prime}\right) \mathrm{d} \vec{X}^{\prime} .
$$

For our purposes, it is desirable to symmetrize $\vec{C}_{a ; b}$ and replace it by the operator

$$
\vec{C}_{(a ; b)}:=\left(0 \ldots 0,\left[\vec{x}_{a-b} ; B_{a b}\right], 0 \ldots 0,\left[\vec{x}_{b-a} ; B_{b a}\right], 0 \ldots 0\right)=\vec{C}_{a, b}+\vec{C}_{b, a} .
$$

Subsequently, we average over all particles to obtain the $3 N$-vector operator

$$
\vec{C}:=\sum_{a<b} \vec{C}_{(a ; b)} .
$$


We use this $\vec{C}$ to construct an appropriate action $L(t)$ whose evolution paves the way to estimate (3.1). With this goal in mind, let

$$
\vec{P}:=\left\{p_{j}^{a}\right\}_{j=1,2,3}^{a=1, \ldots, N}
$$

be the overall momentum vector and $C_{a}^{j}$ denote the components of $\vec{C}$. Accordingly, we consider the action

$$
L(t):=\langle\vec{C} \cdot \vec{P}(t) \mid \rho(t)\rangle .
$$

The time evolution of $L(t)$ is described by

$$
\dot{L}(t)=\left\langle\vec{C} \cdot \partial_{t} \vec{P}(t) \mid \rho(t)\right\rangle-\left\langle\vec{P}(t) \mid \vec{C} \partial_{t} \rho(t)\right\rangle .
$$

By using the conservation laws (3.9) and (3.10) and transferring the operator $\vec{C} \nabla$ on the right-hand side of the inner product, we obtain the equation

$$
\dot{L}(t)=S_{\mathrm{cm}}+S_{\mathrm{cv}}+S_{\mathrm{ds}}+S_{\mathrm{pr}} .
$$

The terms $S_{\ell}(\ell=\mathrm{cm}, \mathrm{cv}, \mathrm{ds}, \mathrm{pr})$ on the right-hand side express distinct physical effects and are defined by

$$
\begin{aligned}
S_{\mathrm{cm}} & :=\left\langle\rho^{-1} \nabla_{j}^{a} \rho \nabla_{k}^{b} \rho \mid\left(\nabla_{k}^{b} C_{a}^{j}\right) \rho\right\rangle, \\
S_{\mathrm{cv}} & :=\left\langle\rho^{-1} p_{j}^{a} p_{b}^{k} \mid\left(\nabla_{k}^{b} C_{a}^{j}\right) \rho\right\rangle-\left\langle p_{b}^{k} \mid\left(\nabla_{k}^{b} C_{a}^{j}\right) p_{j}^{a}\right\rangle, \\
S_{\mathrm{ds}} & :=\langle(\operatorname{div} \vec{C})(-\Delta \rho) \mid \rho\rangle, \\
S_{\mathrm{pr}} & :=\langle\vec{C} \cdot(\vec{M} \rho) \mid \rho\rangle .
\end{aligned}
$$

The origin of these terms is described as follows. The term $S_{\mathrm{cm}}$ is due to the compressibility of the fluid described by the conservation laws; $S_{\mathrm{cv}}$ is a convective term; $S_{\mathrm{ds}}$ is due to dispersion; and $S_{\mathrm{pr}}$ signifies a pressure contribution.

Our next goal is to show that all terms $S_{\ell}$ in $(3.21)-(3.24)$ are positive. In particular, $S_{\mathrm{ds}}$ reduces to the integral appearing in (3.1). First, we derive alternative expressions for $S_{\mathrm{cm}}$ and $S_{\mathrm{cv}}$ in terms of appropriate tensor products (see (3.35) and $(3.36))$. To this end, we introduce some additional formulas which will be used below. Recall definition $(3.16)$ and write $\vec{C}=\left(\vec{C}_{1}, \vec{C}_{2}, \ldots, \vec{C}_{N}\right)$. The component $\vec{C}_{a}$ of $\vec{C}$ reads

$$
\vec{C}_{a}=\sum_{b, b \neq a}\left[\vec{x}_{a-b} ; B_{a b}\right]
$$

and has derivative

$$
\nabla^{c} \vec{C}_{a}=\sum_{b, b \neq a}\left(\nabla^{a-b}\left[\vec{x}_{a-b} ; B_{a b}\right]\right)\left(\delta_{a}^{c}-\delta_{b}^{c}\right) .
$$

The combination of the identity

$$
\nabla_{k}^{a-b}\left[x_{a-b}^{j} ; B_{a b}\right]=\delta_{k}^{j} B_{a b}+\left[x_{a-b}^{j} ;\left(\nabla_{k}^{a-b} B_{a b}\right)\right]
$$


with the formula

$$
\begin{aligned}
& \left(\left[x_{a-b}^{j} ; \nabla_{k}^{a-b} B_{a b}\right] f\right)\left(\vec{x}_{a-b}\right) \\
& \quad=-\int_{\mathbb{R}^{3 N}} \frac{\left(x_{a-b}^{j}-x_{a-b}^{\prime, j}\right)\left(x_{k, a-b}-x_{k, a-b}^{\prime}\right)}{\left|\vec{x}_{a-b}-\vec{x}_{a-b}^{\prime}\right|^{3}} f\left(\vec{X}^{\prime}\right) \mathrm{d} \vec{X}^{\prime}
\end{aligned}
$$

yields the expression

$$
\left(\nabla_{k}^{a-b}\left[x_{a-b}^{j} ; B_{a b}\right] f\right)\left(\vec{x}_{a-b}\right)=\int_{\mathbb{R}^{3 N}} r_{k}^{j}\left(\vec{x}_{a-b} ; \vec{x}_{a-b}^{\prime}\right) f\left(\vec{X}^{\prime}\right) \mathrm{d} \vec{X}^{\prime},
$$

where the kernel $r_{k}^{j}$ is defined by

$$
\begin{aligned}
r_{k}^{j}\left(\vec{x}_{a-b} ; \vec{x}_{a-b}^{\prime}\right):= & \left|\vec{x}_{a-b}-\vec{x}_{a-b}^{\prime}\right|^{-3} \\
& \times\left[\left|\vec{x}_{a-b}-\vec{x}_{a-b}^{\prime}\right|^{2} \delta_{k}^{j}-\left(x_{k, a-b}-x_{k, a-b}^{\prime}\right)\left(x_{a-b}^{j}-x_{a-b}^{\prime, j}\right)\right] .
\end{aligned}
$$

This kernel is positive definite. By the usual tensor convention we have

$$
\mathbf{r}_{a b}:=\left(r_{k}^{j}\left(\vec{x}_{a-b} ; \vec{x}_{a-b}^{\prime}\right)\right), \quad j, k=1,2,3 .
$$

With regard to the momentum vector $\vec{P}$ we write $\vec{P}=\left(\vec{p}^{1}, \vec{p}^{2}, \ldots, \vec{p}^{N}\right)$ and denote the tensor product of two momentum components by

$$
\vec{p}^{a} \otimes \vec{p}^{b}=\left(p_{j}^{a} p_{k}^{b}\right), \quad j, k=1,2,3
$$

For a pair $(a, b)$ of particles, we form the linear combinations

$$
\vec{p}^{a \pm b}:=\frac{1}{\sqrt{2}}\left(\vec{p}^{a} \pm \vec{p}^{b}\right), \quad a, b=1,2, \ldots, N,
$$

which correspond to the center-of-mass coordinates (3.3). Consequently, we find the useful relation

$$
\vec{p}^{a-b} \otimes \vec{p}^{a-b}=\frac{1}{2}\left(\vec{p}^{a} \otimes \vec{p}^{a}+\vec{p}^{b} \otimes \vec{p}^{b}-\vec{p}^{a} \otimes \vec{p}^{b}-\vec{p}^{b} \otimes \vec{p}^{a}\right) .
$$

By combining (3.26)-(3.34), we express $S_{\mathrm{cm}}$ and $S_{\mathrm{cv}}$ from (3.21) and (3.22) as

$$
\begin{aligned}
S_{\mathrm{cm}} & =\left\langle\rho^{-1} \nabla \rho \otimes \nabla \rho \mid(\nabla \vec{C}) \rho\right\rangle, \\
S_{\mathrm{cv}} & =\left\langle\rho^{-1} \vec{P} \otimes \vec{P} \mid(\nabla \vec{C}) \rho\right\rangle-\langle\vec{P} \mid(\nabla \vec{C}) \vec{P}\rangle .
\end{aligned}
$$

With (3.23), (3.24), (3.35) and (3.36) in mind, we now show that the terms $S_{\mathrm{cm}}, S_{\mathrm{cv}}, S_{\mathrm{ds}}$ and $S_{\mathrm{pr}}$ entering (3.20) are all positive. In particular, the dispersive term $S_{\mathrm{ds}}$ admits a space integral representation directly related to the left-hand side of (3.1). 
First, we focus on the convective term, $S_{\mathrm{cv}}$. Since the tensor $\mathbf{r}\left(\vec{x}_{a-b} ; \vec{x}_{a-b}^{\prime}\right)$ of (3.31) is evaluated at two points, we form the associated two-point momentum

$$
\vec{J}\left(\vec{X} ; \vec{X}^{\prime}\right):=\sqrt{\rho^{\prime} / \rho} \vec{P}-\sqrt{\rho / \rho^{\prime}} \vec{P}^{\prime},
$$

where

$$
\begin{aligned}
& \vec{P}:=\vec{P}\left(\vec{x}_{a+b}, \vec{x}_{a-b}, \vec{X}_{a, b}\right), \quad \vec{P}^{\prime}=\vec{P}\left(\vec{x}_{a+b}^{\prime}, \vec{x}_{a-b}^{\prime}, \vec{X}_{a, b}^{\prime}\right), \\
& \rho:=\rho\left(\vec{x}_{a+b}, \vec{x}_{a-b}, \vec{X}_{a, b}\right), \quad \rho^{\prime}=\rho\left(\vec{x}_{a+b}^{\prime}, \vec{x}_{a-b}^{\prime}, \vec{X}_{a, b}^{\prime}\right) .
\end{aligned}
$$

Accordingly, the convective term of (3.36) is expressed as the sum

$$
S_{\mathrm{cv}}=2 \sum_{a \neq b} W_{a b}
$$

where the integrals $W_{a b}$ are given by

$$
W_{a b}:=\int \mathrm{d} \vec{x}_{a+b} \mathrm{~d} \vec{x}_{a+b}^{\prime} \mathrm{d} \vec{X}_{a, b} \mathrm{~d} \vec{X}_{a, b}^{\prime}\left\{\left\langle\vec{J}^{a-b} \otimes \vec{J}^{a-b} \mid \mathbf{r}_{a b}\right\rangle_{a-b}\right\} \geq 0,
$$

with the obvious convention

$$
\vec{J}^{a \pm b}:=\frac{1}{\sqrt{2}}\left(\vec{J}^{a} \pm \vec{J}^{b}\right) .
$$

In $(3.41),\langle\cdot \mid \cdot\rangle_{a-b}$ denotes the expectation value, or average, over the variables $\left(\vec{x}_{a-b}, \vec{x}_{a-b}^{\prime}\right)$. By $(3.40)$ and (3.41), we conclude that

$$
S_{\mathrm{cv}} \geq 0 \text {. }
$$

Second, we concentrate on the compressible term, $S_{\mathrm{cm}}$, from (3.35). By introducing the gradient operator

$$
\nabla^{a \pm b}=\frac{1}{\sqrt{2}}\left(\nabla^{a} \pm \nabla^{b}\right)
$$

$S_{\mathrm{cm}}$ is recast to the form

$$
S_{\mathrm{cm}}=2 \sum_{a \neq b}\left\langle\rho^{-1} \nabla^{a-b} \rho \otimes \nabla^{a-b} \rho \mid\left(\mathbf{r}_{a b}\right) \rho\right\rangle .
$$

Without further ado, we conclude that

$$
S_{\mathrm{cm}} \geq 0 \text {. }
$$

Third, we show that the pressure term, $S_{\mathrm{pr}}$, is also positive. This case is more demanding but crucial since it is connected intimately with the nature of particle interactions. Equation (3.24) is recast to the expression

$$
S_{p r}=\sum_{a}\left\langle\vec{C}_{a} \cdot\left(\vec{M}^{a} \rho\right) \mid \rho\right\rangle=\sum_{\substack{a, b, c \\ b \neq a, c \neq a}} Q_{a, b, c},
$$

where the triple interaction terms $Q_{a, b, c}$ have the integral representation

$$
Q_{a, b, c}:=\int w_{c a} \frac{\vec{x}_{c}-\vec{x}_{a}}{\left|\vec{x}_{c}-\vec{x}_{a}\right|} \cdot \frac{\vec{x}_{b}-\vec{x}_{a}-\left(\vec{x}_{b}^{\prime}-\vec{x}_{a}^{\prime}\right)}{\left|\vec{x}_{b}-\vec{x}_{a}-\left(\vec{x}_{b}^{\prime}-\vec{x}_{a}^{\prime}\right)\right|} \rho(\vec{X}) \rho\left(\vec{X}^{\prime}\right) \mathrm{d} \vec{X} \mathrm{~d} \vec{X}^{\prime} .
$$


Recall that $w_{c a}$ is defined in (2.15). To derive the desired estimate, we pair appropriately terms participating in the sum of (3.47). So, we pair the terms

$$
\begin{aligned}
& w_{c a} \frac{\vec{x}_{c}-\vec{x}_{a}}{\left|\vec{x}_{c}-\vec{x}_{a}\right|} \cdot \frac{\vec{x}_{b}-\vec{x}_{a}-\vec{x}_{b}{ }^{\prime}+\vec{x}_{a}^{\prime}}{\left|\vec{x}_{b}-\vec{x}_{a}-\vec{x}_{b}{ }^{\prime}+\vec{x}_{a}^{\prime}\right|} \rho(\vec{X}) \rho\left(\vec{X}^{\prime}\right), \\
& w_{a c} \frac{\vec{x}_{c}-\vec{x}_{a}}{\left|\vec{x}_{c}-\vec{x}_{a}\right|} \cdot \frac{\vec{x}_{b}-\vec{x}_{c}-\vec{x}_{b}{ }^{\prime}+\vec{x}_{c}^{\prime}}{\left|\vec{x}_{b}-\vec{x}_{c}-\vec{x}_{b}{ }^{\prime}+\vec{x}_{c}^{\prime}\right|} \rho(\vec{X}) \rho\left(\vec{X}^{\prime}\right) .
\end{aligned}
$$

In the spirit of Sec. 2, we interpret the above terms geometrically by considering the triangles

$$
\begin{aligned}
& T\left(\vec{x}_{a}, \vec{x}_{c}, \vec{x}_{b}-\vec{x}_{b}{ }^{\prime}+\vec{x}_{a}^{\prime}\right)=: T\left(a, c, b-b^{\prime}+a^{\prime}\right), \\
& T\left(\vec{x}_{a}, \vec{x}_{c}, \vec{x}_{b}-\vec{x}_{b}{ }^{\prime}+\vec{x}_{c}^{\prime}\right)=: T\left(a, c, b-b^{\prime}+c^{\prime}\right),
\end{aligned}
$$

as shown in Fig. 2. By symmetry relation (3.8) for Bosons, we have

$$
\rho\left(\ldots \vec{x}_{a}^{\prime} \ldots \vec{x}_{c}^{\prime} \ldots\right)=\rho\left(\ldots \vec{x}_{c}^{\prime} \ldots \vec{x}_{a}^{\prime} \ldots\right) .
$$

Hence, we can add to (3.49) and (3.50) the contributions from the terms

$$
\begin{aligned}
& w_{c a} \frac{\vec{x}_{c}-\vec{x}_{a}}{\left|\vec{x}_{c}-\vec{x}_{a}\right|} \cdot \frac{\vec{x}_{b}-\vec{x}_{a}-\vec{x}_{b}{ }^{\prime}+\vec{x}_{c}^{\prime}}{\left|\vec{x}_{b}-\vec{x}_{a}-\vec{x}_{b}^{\prime}+\vec{x}_{c}^{\prime}\right|} \rho(\vec{X}) \rho\left(\vec{X}^{\prime \prime}\right), \\
& w_{a c} \frac{\vec{x}_{c}-\vec{x}_{a}}{\left|\vec{x}_{c}-\vec{x}_{a}\right|} \cdot \frac{\vec{x}_{b}-\vec{x}_{c}-\vec{x}_{b}^{\prime}+\vec{x}_{a}^{\prime}}{\left|\vec{x}_{b}-\vec{x}_{c}-\vec{x}_{b}^{\prime}+\vec{x}_{a}^{\prime}\right|} \rho(\vec{X}) \rho\left(\vec{X}^{\prime \prime}\right),
\end{aligned}
$$

where $\vec{X}^{\prime \prime}$ denotes the variable resulting after we switch $\vec{x}_{a}^{\prime}$ and $\vec{x}_{c}{ }^{\prime}$. Accordingly, we consider again the triangles

$$
T\left(a, c, b-b^{\prime}+c^{\prime}\right), \quad T\left(a, c, b-b^{\prime}+a^{\prime}\right) .
$$

Thus, by the notation of Sec. 2, the sum of the four terms described in $(3.49),(3.50),(3.53)$ and $(3.54)$ equals

$$
\begin{aligned}
& w_{c a}\left\{\cos \alpha\left(a, c, b-b^{\prime}+a^{\prime}\right)+\cos \alpha\left(c, a, b-b^{\prime}+c^{\prime}\right)\right\} \\
& \quad+w_{a c}\left\{\cos \alpha\left(a, c, b-b^{\prime}+c^{\prime}\right)+\cos \alpha\left(c, a, b-b^{\prime}+c^{\prime}\right)\right\} ;
\end{aligned}
$$

see Fig. 2. Evidently, this term is positive. Hence, we conclude that

$$
S_{\mathrm{pr}} \geq 0
$$

We now focus on the dispersive term, $S_{\mathrm{ds}}$, and invoke the Fourier transform with respect to the variables $\left\{\vec{x}_{a}\right\}$. Let $\vec{\xi}_{a}$ be the dual variable corresponding to the three-vector $\vec{x}_{a}$. By virtue of the dual $3 N$-vector

$$
\vec{\Xi}=\left(\vec{\xi}_{a+b}, \vec{\xi}_{a-b}, \vec{\Xi}_{a, b}\right),
$$

the Fourier transform of the $3 N$-dimensional Laplacian reads

$$
\mathcal{F}\{-\boldsymbol{\Delta}\}=\left|\vec{\xi}_{a+b}\right|^{2}+\left|\vec{\xi}_{a-b}\right|^{2}+\left|\vec{\Xi}_{a, b}\right|^{2} .
$$




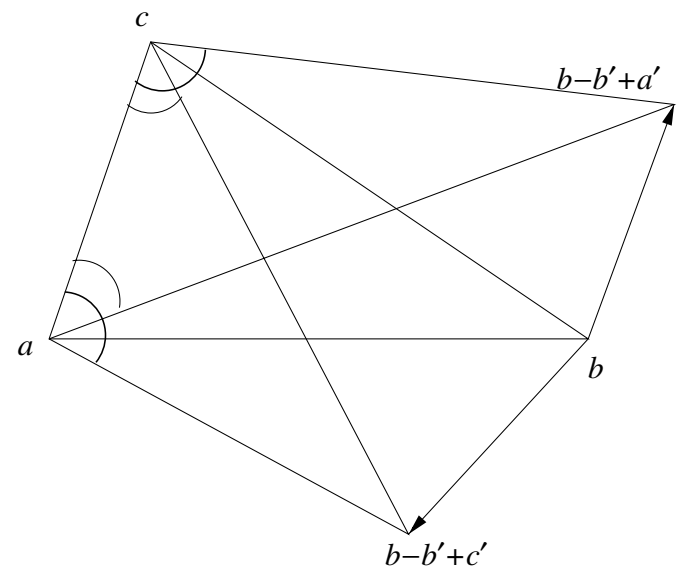

Fig. 2. Geometric interpretation of interaction terms from (3.47) and (3.48).

Note that formula (3.23) for $S_{\mathrm{ds}}$ involves $\operatorname{div} \vec{C}$. By (3.12)-(3.16), the divergence of $\vec{C}$ is

$$
\operatorname{div} \vec{C}=2 \sum_{a \neq b} B_{a b}
$$

In view of (3.12), the Fourier transform of the requisite $B_{a b}$ is

$$
\mathcal{F}\left\{B_{a b}\right\}=\frac{\delta\left(\vec{\xi}_{a+b}\right) \delta\left(\vec{\Xi}_{a, b}\right)}{\left|\vec{\xi}_{a-b}\right|^{2}} .
$$

Thus, we readily compute the transform

$$
\mathcal{F}\{\operatorname{div} \vec{C}(-\boldsymbol{\Delta})\}=\sum_{a \neq b} \mathbf{1}\left(\vec{\xi}_{a-b}\right) \delta\left(\vec{\xi}_{a+b}\right) \delta\left(\vec{\Xi}_{a, b}\right) .
$$

By expressing $S_{\mathrm{ds}}$ in terms of Fourier transforms, we conclude that the dispersive term is in fact the integral

$$
S_{\mathrm{ds}}=\sum_{a \neq b} \int_{\mathbb{R}^{3}} \mathrm{~d} \vec{x}_{a-b}\left\{\left(\widetilde{\rho}_{a b}\right)^{2}\right\},
$$

where the reduced density $\widetilde{\rho}$ is introduced in (3.2).

So far, we proved that the terms entering the right-hand side of (3.19), the evolution equation for $L(t)$, are positive. In particular, $S_{\mathrm{ds}}$ is described by integral (3.62). The final stage of the proof involves integrating (3.20) over a time interval $[0, T]$. Thus, we obtain the equation

$$
\int_{0}^{T} \mathrm{~d} t\left\{S_{\mathrm{cm}}+S_{\mathrm{cv}}+S_{\mathrm{ds}}+S_{\mathrm{pr}}\right\}=L(T)-L(0) .
$$

Furthermore, the action $L(t)$ defined in (3.18) is bounded by $\left\|\psi_{N}\right\|_{H^{1}}\left\|\psi_{N}\right\|_{L^{2}}^{3}$. Consequently, we reach the desired space-time estimate (3.1). Note that the right-hand 
side of (3.1) is bounded by the energy $E_{N}(t)$ of the system; cf. (2.41). This statement concludes the proof.

Estimate (3.1) can be modified to become (3.5) by means exactly analogous to those developed in Sec. 2. For initial data that form a tensor product, we have the bound

$$
\sum_{a \neq b} \int_{\mathbb{R} \times \mathbb{R}^{3}} \mathrm{~d} t \mathrm{~d} \vec{x}_{a-b}\left\{\left(\widetilde{\rho}_{a, b}\left(t, \vec{x}_{a b}\right)\right)^{2}\right\} \leq N^{2} \sqrt{E_{1}}\left\|\psi_{N}\right\|_{L^{2}}^{3},
$$

which is manifestly independent of the number, $N$, of particles.

We close this section with a few comments. By defining the function

$$
D\left(\vec{X} ; \vec{X}^{\prime}\right):=\sum_{a<b}\left|\vec{x}_{a-b}-\vec{x}_{a-b}^{\prime}\right|
$$

and the correlation function

$$
\mathcal{C}(t):=\int \mathrm{d} \vec{X} \mathrm{~d} \vec{X}^{\prime}\left\{D\left(\vec{X} ; \vec{X}^{\prime}\right) \rho(t, \vec{X}) \rho\left(t, \vec{X}^{\prime}\right)\right\},
$$

we deduce the relations

$$
\dot{\mathcal{C}}(t)=L(t), \quad \dot{L}(t) \geq 0 .
$$

Because $L(t)$ is increasing and bounded by the total energy $E_{N}(t)$, we conclude that

$$
\lim _{t \rightarrow \pm \infty} L(t)=L_{ \pm}
$$

i.e. $\mathcal{C}(t)$ in $(3.66)$ is a convex function with a unique minimum, and is asymptotically linear at $t= \pm \infty$.

\section{Correlations and BBGKY Hierarchy}

This section has two parts. The first part serves a brief review of the description of the many-body Hamiltonian evolution for Bosons in terms of a BBGKY hierarchy for particle reduced density matrices $[7-10,26,27]$. In the second part, we derive an estimate for the one-particle marginal $\gamma_{1}$; see (4.17) and (4.20).

\subsection{Review of BBGKY hierarchy for $k$-particle marginals}

We start again with the $N$-body Schrödinger equation (3.6). The ultimate goal is to investigate aspects of this evolution for large $N$. Following EESY [7-10], we apply the idea that the two-body interaction $V$ is scaled in some way by incorporating the notion that pair collisions are relatively rare and weak. One particular scaling for $V$ is $[7-10]$

$$
V\left(\left|\vec{x}_{a}-\vec{x}_{b}\right|\right)=N^{2} \mathcal{V}_{1}\left(N\left|\vec{x}_{a}-\vec{x}_{b}\right|\right)=\frac{1}{N}\left\{N^{3} V_{1}\left(N\left|\vec{x}_{a}-\vec{x}_{b}\right|\right)\right\},
$$

which is equivalent to (1.6) with a scattering length $l=O\left(N^{-1}\right)$. The scaling with $N$ is chosen so that the quantity inside the curly brackets approaches a constant 
times the delta function as $N \rightarrow \infty$. We remind the reader that because of Boson symmetry the $N$-body wave function $\psi_{N}$ is invariant under permutations of the space variables, i.e.

$$
\psi_{N}\left(t, \vec{x}_{1}, \vec{x}_{2}, \ldots, \vec{x}_{N}\right)=\psi_{N}\left(t, \vec{x}_{\pi(1)}, \vec{x}_{\pi(2)}, \ldots, \vec{x}_{\pi(N)}\right)
$$

where $\pi(a)$ is a permutation of the indices $\{1,2, \ldots, N\}$. Although we are interested in the time evolution of the system, for the sake of convenience we will omit the time $(t)$ dependence whenever this is not relevant to the discussion.

A description of the particle system via a BBGKY hierarchy was proposed by Spohn $[26,27]$ and forms the starting point in the analysis by EESY [7-10]. A key element of this methodology is the density matrix $\gamma_{N}:=\left|\psi_{N}\right\rangle\left\langle\psi_{N}\right|$, which is a trace class operator represented by

$$
\gamma_{N}\left(t, \vec{x}_{1}, \ldots, \vec{x}_{N} \mid \vec{x}_{1}^{\prime}, \ldots, \vec{x}_{N}^{\prime}\right):=\psi_{N}\left(t, \vec{x}_{1}, \ldots, \vec{x}_{N}\right) \psi_{N}^{*}\left(t, \vec{x}_{1}^{\prime}, \ldots, \vec{x}_{N}^{\prime}\right)
$$

By virtue of (3.6), the evolution equation for $\gamma_{N}$ is

$$
i \partial_{t} \gamma_{N}+\left(-\boldsymbol{\Delta}_{N}+\boldsymbol{\Delta}_{N^{\prime}}\right) \gamma_{N}+\sum_{a \neq b, a^{\prime} \neq b^{\prime}}\left(V_{a b}-V_{a^{\prime} b^{\prime}}\right) \gamma_{N}=0
$$

where $\boldsymbol{\Delta}_{N}=\sum_{a} \Delta_{a}$ denotes the $3 N$-dimensional Laplacian, $V_{a b}:=V\left(\left|\vec{x}_{a}-\vec{x}_{b}\right|\right)$, and the primed indices indicate the coordinates $\left(\vec{x}_{1}^{\prime} \ldots \vec{x}_{N}^{\prime}\right)$.

Next, we describe features of the $k$-particle marginals $\gamma_{k}$ stemming from $\gamma_{N}$. For this purpose, it is convenient to define $\vec{X}_{N}:=\left(\vec{x}_{1}, \vec{x}_{2}, \ldots, \vec{x}_{N}\right)$ and $\vec{X}_{N}^{\prime}:=$ $\left(\vec{x}_{1}^{\prime}, \ldots, \vec{x}_{N}^{\prime}\right)$. In order to average over some of the coordinates, we also define [7-10]

$$
\vec{X}_{k}=\left(\vec{x}_{1}, \ldots, \vec{x}_{k}\right), \quad \vec{X}_{N-k}=\left(\vec{x}_{k+1}, \ldots, \vec{x}_{N}\right)
$$

thus, $\vec{X}_{N}=\left(\vec{X}_{k}, \vec{X}_{N-k}\right)$ for $k=1,2, \ldots, N$. The $k$-particle marginals as formed via the partial averaging

$$
\gamma_{k}\left(\vec{X}_{k} \mid \vec{X}_{k}^{\prime}\right)=\int \mathrm{d} \vec{Y}_{N-k}\left\{\gamma_{N}\left(\vec{X}_{k}, \vec{Y}_{N-k} \mid \vec{X}_{k}^{\prime}, \vec{Y}_{N-k}\right)\right\}
$$

Note that because of (4.2) it does not matter which variables we average out.

We now describe the evolution law for $\gamma_{k}$ using (4.4) and definition (4.6). By (3.3), let $\nabla_{a \pm b}$ be the gradient operator corresponding to $\vec{x}_{a \pm b}$. In view of $\Delta_{a}-\Delta_{a^{\prime}}=\nabla_{a+a^{\prime}} \cdot \nabla_{a-a^{\prime}}$, setting $a=a^{\prime}$ and integrating over $a+a^{\prime}$ for the averaged variables $\gamma_{k}$ yields zero. Define the sets $J_{k}:=\{1,2, \ldots, k\}, J_{k}^{\prime}:=\left\{1^{\prime}, 2^{\prime}, \ldots, k^{\prime}\right\}$ and $J_{k}^{c}=\{k+1, \ldots, N\}$, the complement of $J_{k}$. The above averaging also produces zero for the potentials $V_{a b}$ and $V_{a^{\prime} b^{\prime}}$ if $a, b \in J_{k}^{c}$ or $a^{\prime}, b^{\prime} \in J_{k}^{c \prime}$ when we identify the variables $a=a^{\prime}$ and $b=b^{\prime}$, i.e. we identify the coordinates $\left(\vec{x}_{k+1}, \ldots, \vec{x}_{N}\right)$ with 
$\left(\vec{x}_{k+1}^{\prime}, \ldots, \vec{x}_{N}^{\prime}\right)$. Consequently, the evolution equation for $\gamma_{k}$ reads

$$
\begin{aligned}
i \partial_{t} \gamma_{k}- & \left(\boldsymbol{\Delta}_{\mathbf{k}}-\boldsymbol{\Delta}_{k}^{\prime}\right) \gamma_{k}+\left(\sum_{\substack{a, b \in J_{k} \\
a \neq b}} V_{a b}-\sum_{\substack{a^{\prime}, b^{\prime} \in J_{k}^{\prime} \\
a^{\prime} \neq b^{\prime}}} V_{a^{\prime} b^{\prime}}\right) \gamma_{k} \\
& +\sum_{a \in J_{k}, a^{\prime} \in J_{k}^{\prime}} \sum_{b \in J_{k}^{c}} \int \mathrm{d} \vec{Y}_{N-k}\left[V\left(\left|\vec{x}_{a}-\vec{y}_{b}\right|\right)-V\left(\left|\vec{x}_{a^{\prime}}-\vec{y}_{b}\right|\right)\right] \\
& \times \gamma_{N}\left(\vec{x}_{1} \ldots \vec{x}_{a} \ldots \vec{x}_{k}, \vec{y}_{k+1} \ldots \vec{y}_{b} \ldots \vec{y}_{N} \mid \vec{x}_{1}^{\prime} \ldots \vec{x}_{a}^{\prime} \ldots \vec{x}_{k}^{\prime}, \vec{y}_{k+1} \ldots \vec{y}_{b} \ldots \vec{y}_{N}\right) \\
= & 0 .
\end{aligned}
$$

We proceed to write (4.7) as a BBGKY-type hierarchy for $\gamma_{k}$. Because of Boson symmetry, integrations over $b \in J_{k}^{c}$ are reduced to

$$
\begin{aligned}
& (N-k) \sum_{a \in J_{k}} \int \mathrm{d} \vec{Y}_{N-k-1} \mathrm{~d} \vec{y}_{k+1}\left[V\left(\left|\vec{x}_{a}-\vec{y}_{k+1}\right|\right)-V\left(\left|\vec{x}_{a}^{\prime}-\vec{y}_{k+1}\right|\right)\right] \\
& \quad \times \gamma_{N}\left(\vec{x}_{1} \ldots \vec{x}_{a} \ldots \vec{x}_{k}, \vec{y}_{k+1}, \vec{Y}_{N-k-1} \mid \vec{x}_{1}^{\prime} \ldots \vec{x}_{a}^{\prime} \ldots \vec{x}_{k}^{\prime}, \vec{y}_{k+1}, \vec{Y}_{N-k-1}\right) .
\end{aligned}
$$

With the definitions $H_{k}:=-\boldsymbol{\Delta}_{k}+\sum_{a, b \in J_{k}} V_{a b}$ where $a \neq b$ and

$$
\begin{aligned}
\mathcal{C}_{V, k+1}^{a, a^{\prime}}\left[\gamma_{k+1}\right]= & \left.\int \mathrm{d} \vec{y}_{k+1}\left[V\left(\left|\vec{x}_{a}-\vec{y}_{k+1}\right|\right)-V\left(\left|\vec{x}_{a}^{\prime}-\vec{y}_{k+1}\right|\right)\right]\right) \\
& \times \gamma_{k+1}\left(X_{k}, y_{k+1} \mid X_{k}^{\prime}, y_{k+1}\right),
\end{aligned}
$$

(4.7) takes the form

$$
i \partial_{t} \gamma_{k}+\left(H_{k}-H_{k^{\prime}}\right) \gamma_{k}+(N-k) \sum_{a \in J_{k}} \sum_{a^{\prime} \in J_{k}^{\prime}} \mathcal{C}_{V, k+1}^{a, a^{\prime}}\left[\gamma_{k+1}\right]=0
$$

This set of coupled of equations constitutes a finite BBGKY-type hierarchy for $\gamma_{k}$ where $k=1,2, \ldots, N$. Evidently, each $\gamma_{k}$ depends on $N$.

\subsection{Estimate for one-particle density matrix $\gamma_{1}$}

So far, we have essentially reviewed the formulation by EESY [7-10]. In the following, we exploit this framework to derive an estimate that involves $\gamma_{1}$. We assume that the interaction potential $V_{1}$ of (1.6) is integrable, $V_{1} \in L^{1}\left(\mathbb{R}^{3}\right)$.

For finite $N$, the first equation of hierarchy (4.10) reads

$$
i \partial_{t} \gamma_{1}-\left(\Delta_{1}-\Delta_{1^{\prime}}\right) \gamma_{1}+(N-1) \mathcal{C}_{V, 2}^{1,1^{\prime}}\left[\gamma_{2}\right]=0
$$

where $\gamma_{2}=\gamma_{2}\left(\vec{x}_{1}, \vec{x}_{2} \mid \vec{x}_{1}^{\prime}, \vec{x}_{2}^{\prime}\right)$. To motivate the analysis for finite $N$ given below, we first consider the simpler case where $N \rightarrow \infty$, in which the interaction potential 
approaches a constant times a delta function. As $N \rightarrow \infty$, by the scaling of (4.1), we have the limit

$$
V_{N}(\cdot):=(N-1) N^{2} V_{1}\left(N\left|\vec{x}_{a}-\vec{y}_{k+1}\right|\right) \rightarrow g \delta\left(\left|\vec{x}_{a}-\vec{y}_{k+1}\right|\right),
$$

where $g$ is a fixed positive parameter $(g>0)$. In effect, we arrive at the basic collapse mechanism that reduces $\gamma_{2}$ to a function of 6 variables, viz.

$$
\mathcal{B}_{2}\left[\gamma_{2}\right]:=g\left\{\gamma_{2}\left(\vec{x}_{1}, \vec{x}_{1} \mid \vec{x}_{1}^{\prime}, \vec{x}_{1}\right)-\gamma_{2}\left(\vec{x}_{1}, \vec{x}_{1}^{\prime} \mid \vec{x}_{1}^{\prime}, \vec{x}_{1}^{\prime}\right)\right\} .
$$

Thus, (4.11) becomes

$$
i \partial_{t} \gamma_{1}-\left(\Delta_{1}-\Delta_{1^{\prime}}\right) \gamma_{1}+\mathcal{B}_{2}\left[\gamma_{2}\right]=0
$$

The partial Fourier transform of (4.14) has the relatively simple structure of a transport law (see (4.18)). Next, we derive an estimate for the forcing term $\mathcal{B}_{2}\left[\gamma_{2}\right]$ of (4.14). Notice that the operator $\mathcal{B}_{2}$ acting on $\gamma_{2}$ is written as

$$
\begin{aligned}
\mathcal{B}_{2}\left[\gamma_{2}\right]= & g \int \mathrm{d} \vec{Y}_{N-2}\left\{\psi_{N}\left(\vec{x}_{1}, \vec{x}_{1} ; \vec{Y}_{N-2}\right) \psi_{N}^{*}\left(\vec{x}_{1}, \vec{x}_{1}^{\prime} ; \vec{Y}_{N-2}\right)-\psi_{N}\left(\vec{x}_{1}, \vec{x}_{1}^{\prime} ; \vec{Y}_{N-2}\right)\right. \\
& \left.\times \psi_{N}^{*}\left(\vec{x}_{1}^{\prime}, \vec{x}_{1}^{\prime} ; \vec{Y}_{N-2}\right)\right\} .
\end{aligned}
$$

By writing

$$
\widetilde{\rho}\left(\vec{x}_{1}, \vec{x}_{2}\right):=\int \mathrm{d} \vec{Y}_{N-2}\left\{\frac{1}{2}\left|\psi_{N}\left(\vec{x}_{1}, \vec{x}_{2}, \vec{Y}_{N-2}\right)\right|^{2}\right\}
$$

and denoting $\mathcal{B}_{2}\left[\gamma_{2}\right]$ by $\mathcal{B}_{2}\left(\vec{x}_{1}, \vec{x}_{1}^{\prime}\right)$, we have the estimate

$$
\begin{aligned}
\left\|\mathcal{B}_{2}(t)\right\|_{L_{1-1^{\prime}}^{2}\left(L_{1+1^{\prime}}^{1}\right)}^{2} & =\int \mathrm{d} \vec{x}_{1-1^{\prime}}\left[\int \mathrm{d} \vec{x}_{1+1^{\prime}} \mid \mathcal{B}_{2}\left(\vec{x}_{1+1^{\prime}}, \vec{x}_{1-1^{\prime}}\right)\right]^{2} \\
& \leq 2 \int \mathrm{d} \vec{x}_{1-1^{\prime}}\left[\int \mathrm{d} \vec{x}_{1+1^{\prime}}\left\{\sqrt{\widetilde{\rho}}\left(\vec{x}_{1}, \vec{x}_{1}\right) \sqrt{\widetilde{\rho}}\left(\vec{x}_{1}, \vec{x}_{1}^{\prime}\right)\right\}\right]^{2} \\
& \leq\left(\int \mathrm{d} \overrightarrow{x_{1}} \widetilde{\rho}\left(\vec{x}_{1}, \vec{x}_{1}\right)\right)\left[\int \mathrm{d} \vec{x}_{1-1^{\prime}}\left(\int \mathrm{d} \vec{x}_{1+1^{\prime}} \widetilde{\rho}\left(\vec{x}_{1}, \vec{x}_{1}^{\prime}\right)\right)\right] \\
& =c \int \mathrm{d} \vec{x}_{1} \widetilde{\rho}\left(\vec{x}_{1}, \vec{x}_{1}\right),
\end{aligned}
$$

by the definition of $\rho$ and $\widetilde{\rho}$. It should be borne in mind that the density $\widetilde{\rho}$ depends on $t$.

In order to study the implication of (4.17), let us write $\Delta_{1}-\Delta_{1^{\prime}}=\nabla_{1+1^{\prime}} \cdot \nabla_{1-1^{\prime}}$ and $\mathcal{B}_{2}\left(\vec{x}_{1}, \vec{x}_{1^{\prime}}\right)=\mathcal{B}_{2}\left(\vec{x}_{1+1^{\prime}}, \vec{x}_{1-1^{\prime}}\right)$. We proceed to derive an estimate involving $\gamma_{1}$. By taking the Fourier transform of (4.14) in the $\vec{x}_{1+1^{\prime}}$ variable and denoting the dual variable by $\vec{v}$, we arrive at the transport equation

$$
\partial_{t} \widehat{\gamma}_{1}-2 \pi \vec{v} \cdot \nabla_{1-1^{\prime}} \widehat{\gamma}_{1}=\widehat{\mathcal{B}}_{2}\left(t, \vec{v}, \vec{x}_{1-1^{\prime}}\right),
$$


where $\widehat{\gamma}_{1}\left(t, \vec{v}, \vec{x}_{1-1^{\prime}}\right)$ denotes the Fourier-transformed function. For the sake of some simplicity, we assume that the initial data for $\gamma_{1}$ are zero, i.e. $\gamma_{1}(t=0)=0$. Thus, the solution to (4.18) is

$$
\widehat{\gamma}_{1}\left(t, \vec{v}, \vec{x}_{1-1^{\prime}}\right)=\int_{0}^{t} \widehat{\mathcal{B}}_{2}\left(s, \vec{v}, \vec{x}_{1-1^{\prime}}-2 \pi s \vec{v}\right) \mathrm{d} s .
$$

On the basis of (4.19), we derive the estimate

$$
\begin{aligned}
\sup _{t, \vec{v}}\left\|\widehat{\gamma}_{1}\right\|_{L^{2}}^{2} & =\sup _{t, \vec{v}}\left\|\int_{0}^{t} \mathrm{~d} s \int \mathrm{d} \vec{y}\left\{e^{i 2 \pi \vec{y} \cdot \vec{v}} \mathcal{B}_{2}\left(s, \vec{y}, \vec{x}_{1-1^{\prime}}-2 \pi s \vec{v}\right)\right\}\right\|_{L_{1-1^{\prime}}^{2}}^{2} \\
& \leq\left\|\int_{0}^{t} \mathrm{~d} s\right\| \mathcal{B}_{2}(s)\left\|_{L_{1+1^{\prime}}^{1}}\right\|_{L_{1-1^{\prime}}^{2}}^{2} \\
& \leq|t| \int_{0}^{t} \mathrm{~d} s\left\|\mathcal{B}_{2}(s)\right\|_{L_{1-1^{\prime}}^{2}\left(L_{1+1^{\prime}}^{1}\right)}^{2}
\end{aligned}
$$

The combination of (4.20) with (4.17) and (2.1) provides the desired estimate for $\gamma_{1}$ as $N \rightarrow \infty$.

This analysis applies directly to the case of finite $N$ with minor modifications. First, (4.18)-(4.20) remain intact under the replacement $(N-1) \mathcal{C}_{V, 2}^{1,1^{\prime}} \equiv \mathcal{B}_{2}$ in (4.11). Second, in view of Remark 2.3 and for $V_{1} \in L^{1}\left(\mathbb{R}^{3}\right)$, estimate (4.17) becomes

$$
\begin{aligned}
& \left\|\mathcal{B}_{2}(t)\right\|_{L_{1-1^{\prime}}^{2}\left(L_{1+1^{\prime}}^{1}\right)}^{2} \\
& \leq\left(\int \mathrm{d} \vec{d} V_{N}(\vec{d}) \int \mathrm{d} \vec{x}_{1} \widetilde{\rho}\left(\vec{x}_{1}, \vec{x}_{1}+\vec{d}\right)\right)\left[\int \mathrm{d} \vec{x}_{1-1^{\prime}}\left(\int \mathrm{d} \vec{x}_{1+1^{\prime}} \widetilde{\rho}\left(\vec{x}_{1}, \vec{x}_{1}^{\prime}\right)\right)\right]
\end{aligned}
$$

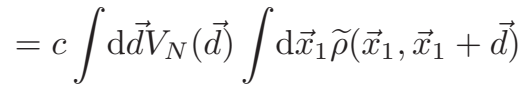

$$
\begin{aligned}
& \leq\left\|V_{N}\right\|_{L^{1}} \sup _{\vec{d}} \int \mathrm{d} \vec{x}_{1} \widetilde{\rho}\left(\vec{x}_{1}, \vec{x}_{1}+\vec{d}\right) .
\end{aligned}
$$

Next, we explain how one obtains estimates for $\gamma_{1}$ that are manifestly independent of the number, $N$, of particles (in the spirit of Secs. 2 and 3). For this purpose, we consider initial data for $\psi_{N}$ that form a tensor product, and retain the potential $V$. Equation (4.11) for finite large $N$ can be written as

$$
i \partial_{t} \gamma_{1}-\left(\Delta_{1}-\Delta_{1^{\prime}}\right) \gamma_{1}+[(N-1) / N] \mathcal{C}_{V}\left[\gamma_{2}\right]=0,
$$

where

$$
\mathcal{C}_{V}\left[\gamma_{2}\right]=\int \mathrm{d} \vec{z} \widetilde{V}_{N}(\vec{z})\left[\gamma\left(\vec{x}_{1}, \vec{x}_{1^{\prime}}-\vec{z} \mid \vec{x}_{1}, \vec{x}_{1^{\prime}}\right)-\gamma_{2}\left(\vec{x}_{1}, \vec{x}_{1^{\prime}} \mid \vec{x}_{1^{\prime}}, \vec{x}_{1^{\prime}}-\vec{z}\right)\right],
$$

and $\widetilde{V}_{N}(\vec{z}):=N^{3} V(N \vec{z})$. This scaled potential is expected to approach a delta function. Thus, any acceptable estimate should involve only $\left\|\widetilde{V}_{N}\right\|_{L^{1}}$, since this is the only $N$-independent quantity. 
Notice that the $\gamma_{2}$ in (4.23) is bounded by the marginal densities of (4.16),

$$
\left|\gamma_{2}\left(t, \vec{x}_{1}, \vec{x}_{2} \mid \vec{x}_{1^{\prime}}, \vec{x}_{2^{\prime}}\right)\right| \leq \sqrt{\rho\left(t, \vec{x}_{1}, \vec{x}_{2}\right)} \sqrt{\rho\left(t, \vec{x}_{1^{\prime}}, \vec{x}_{2^{\prime}}\right)}
$$

Using (4.24), the form of $\mathcal{C}_{V}$ by (4.23) and Cauchy-Schwartz, we find

$$
\begin{aligned}
\int_{0}^{T} \mathrm{~d} t\left\|\mathcal{C}_{V}\left[\gamma_{2}\right]\right\|_{L_{1+1^{\prime}}^{1}} & \leq\left\|\widetilde{V}_{N}\right\|_{L^{1}} \sup _{\vec{z}}\left(\int \mathrm{d} t \mathrm{~d} \vec{x}_{1}\left\{\rho\left(t, \vec{x}_{1}, \vec{x}_{1}-\vec{z}\right)\right\}\right)^{1 / 2} \\
& \times\left(\int_{[0, T] \times \mathbb{R}^{3}} \mathrm{~d} t \mathrm{~d} \vec{x}_{1+1^{\prime}}\left\{\rho\left(t, \vec{x}_{1}, \vec{x}_{1^{\prime}}\right)\right\}\right)^{1 / 2} .
\end{aligned}
$$

The first observation is that the quantity

$$
\int \mathrm{d} t \mathrm{~d} \vec{x}_{1}\left\{\rho\left(t, \vec{x}_{1}, \vec{x}_{1}-\vec{z}\right)\right\}
$$

is estimated by $C \sqrt{E_{1}}$ where $E_{1}$ is the energy per particle $\left(E_{N}(t=0)=N E_{1}\right)$. Therefore, if we square and integrate with respect to the $1-1^{\prime}$ variables, we have

$$
\left\|\int_{0}^{T} \mathrm{~d} t\right\| \mathcal{C}_{V}\left[\gamma_{2}\right]\left\|_{L_{1+1^{\prime}}^{1}}\right\|_{L_{1-1^{\prime}}^{2}}^{2} \leq C T .
$$

The last inequality implies the estimate

$$
\sup _{\vec{v}}\left\|\widehat{\gamma}_{1}(t)\right\|_{L^{2}} \leq \sqrt{|t|} C .
$$

Similarly, if we raise (4.25) to the fourth power and integrate, we obtain

$$
\sup _{\vec{v}}\left\|\widehat{\gamma}_{1}(t)\right\|_{L^{4}} \leq|t|^{1 / 4} C \text {. }
$$

Here, we used

$$
\begin{aligned}
& \int \mathrm{d} \vec{x}_{1-1^{\prime}}\left(\int_{[0, T] \times \mathbb{R}^{3}} \mathrm{~d} t \mathrm{~d} \vec{x}_{1+1^{\prime}} \rho\left(t, \vec{x}_{1}, \vec{x}_{1^{\prime}}\right)\right)^{2} \\
& \leq T \int \mathrm{d} t \mathrm{~d} \vec{x}_{1-1^{\prime}}\left(\int \mathrm{d} \vec{x}_{1+1^{\prime}} \rho\left(t, \vec{x}_{1}, \vec{x}_{1^{\prime}}\right)\right)^{2}
\end{aligned}
$$

along with the fact that the quantity

$$
\int \mathrm{d} t \mathrm{~d} \vec{x}_{1-1^{\prime}}\left(\int \mathrm{d} \vec{x}_{1+1^{\prime}} \rho\left(t, \vec{x}_{1}, \vec{x}_{1}^{\prime}\right)\right)^{2}
$$

is estimated by $C \sqrt{E_{1}}$. The derived estimates for $\gamma_{1}$ are independent of $N$ but do depend on time because we treat $\mathcal{C}_{V}\left[\gamma_{2}\right]$ simply as forcing in the transport equation.

It is natural to ask whether this analysis can be extended to $k$-particle marginals for $k>1$. However, evolution equations (4.10) satisfied by $\gamma_{k}$ cannot be converted to simple transport laws if $k>1$ because of the presence of interaction potentials. Hence, the procedure applied hitherto is not directly applicable to the case with $k>1$. The derivation of estimates for $\gamma_{k>1}$ is left for future work. 


\section{Acknowledgment}

The second author appreciates the support of the Maryland NanoCenter.

\section{References}

[1] F. A. Berezin, Method of Second Quantization (Academic Press, 1966).

[2] J. M. Blatt and V. F. Weisskopf, Theoretical Nuclear Physics (Wiley, 1952), pp. 7476.

[3] E. Calzetta and B.-L. Hu, Gauge-invariant effective action for the dynamics of BoseEinstein condensates with a fixed number of atoms, preprint, cond-mat/0508240.

[4] J. Colliander, M. G. Grillakis and N. Tzirakis, Tensor products and correlation estimates with applications to nonlinear Schrödinger equations, submitted.

[5] J. Colliander, M. Keel, G. Staffilani, K. Takaoka and T. Tao, Generalizations of the interaction Morawetz inequality, preprint.

[6] F. J. Dyson, Ground-state energy of a hard sphere gas, Phys. Rev. 106 (1957) 20-26.

[7] A. Elgart, L. Erdős, B. Schlein and H.-T. Yau, Gross-Pitaevskii equation as the mean field limit of weakly coupled bosons, Arch. Ration. Mech. Anal. 179 (2006) 265-283.

[8] L. Erdős, B. Schlein and H.-T. Yau, Derivation of the Gross-Pitaevskii equation for the dynamics of Bose-Einstein condensate, Commun. Pure Appl. Math. 59 (2006) 1659-1741.

[9] L. Erdős, B. Schlein and H.-T. Yau, Derivation of the cubic non-linear Schrödinger equation from quantum dynamics of many-body systems, Invent. Math. 167 (2007) 515-614.

[10] L. Erdős, B. Schlein and H.-T. Yau, Rigorous derivation of the Gross-Pitaevskii equation, Phys. Rev. Lett. 98 (2007) 040404.

[11] M. G. Grillakis, unpublished.

[12] E. P. Gross, Structure of a quantized vortex in boson systems, Nuovo Cim. 20 (1961) 454-477.

[13] E. P. Gross, Hydrodynamics of a superfluid condensate, J. Math. Phys. 4 (1963) 195-207.

[14] K. Huang and C. N. Yang, Quantum-mechanical many-body problem with hardsphere interaction, Phys. Rev. 105 (1957) 767-775.

[15] K. Huang, C. N. Yang and J. M. Luttinger, Imperfect Bose gas with hard-sphere interaction, Phys. Rev. 105 (1957) 776-784.

[16] S. Klainerman and M. Machedon, On the uniqueness of solutions to the GrossPitaevskii hierarchy, Commun. Math. Phys. 279 (2008) 169-185.

[17] T. D. Lee, K. Huang and C. N. Yang, Eigenvalues and eigengunctions of a Bose system of hard spheres and its low-temperature properties, Phys. Rev. 106 (1957) 1135-1145.

[18] T. D. Lee and C. N. Yang, Many body problem in quantum mechanics and quantum statistical mechanics, Phys. Rev. 105 (1957) 1119-1120.

[19] E. H. Lieb and R. Seiringer, Derivation of the Gross-Pitaevskii equation for rotating Bose gases, Commun. Math. Phys. 264 (2006) 505-537.

[20] E. H. Lieb, R. Seiringer, J. P. Solovej and J. Yngvason, The Mathematics of the Bose Gas and Its Condensation (Birkhäuser, 2005).

[21] E. H. Lieb, R. Seiringer and J. Yngvason, Bosons in a trap: A rigorous derivation of the Gross-Pitaevskii energy functional, Phys. Rev. A 61 (2000) 043602.

[22] E. H. Lieb, R. Seiringer and J. Yngvason, A rigorous derivation of the Gross-Pitaevskii energy functional for a two-dimensional Bose gas, Commun. Math. Phys. 224 (2001) $17-31$. 
[23] J. Lin and W. Strauss, Decay and scattering of solutions of a nonlinear Schrödinger equation, J. Funct. Anal. 30 (1978) 245-263.

[24] D. Margetis, Studies in Classical Electromagnetic Radiation and Bose-Einstein Condensation, Ph.D. thesis, Harvard University (1999).

[25] L. P. Pitaevskii, Vortex lines in an imperfect Bose gas, Soviet Phys. JETP 13 (1961) 451-454.

[26] H. Spohn, Kinetic equations from Hamiltonian dynamics: Markovian limits, Rev. Mod. Phys. 52 (1980) 569-615.

[27] H. Spohn, Large Scale Dynamics of Interacting Particles (Springer Verlag, 1991).

[28] T. Tao, Nonlinear Dispersive Equations: Local and Global Analysis, CBMS Regional Conference Series in Mathematics, No. 106 (American Mathematical Society, 2006).

[29] T. T. Wu, Ground state of a Bose system of hard spheres, Phys. Rev. 115 (1958) 1390-1404.

[30] T. T. Wu, Some nonequilibrium properties of a Bose system of hard spheres at extremely low temperatures, J. Math. Phys. 2 (1961) 105-123.

[31] T. T. Wu, Bose-Einstein condensation in an external potential at zero temperature: General theory, Phys. Rev. A 58 (1998) 1465-1474. 